\title{
The Microscopic Derivation and Well-Posedness of the Stochastic Keller-Segel Equation
}

\author{
Hui Huang ${ }^{1}\left[\right.$ ] Jinniao Qiu ${ }^{2}$ \\ Received: 22 December 2019 / Accepted: 22 October 2020 / Published online: 18 December 2020 \\ (c) The Author(s) 2020
}

\begin{abstract}
In this paper, we propose and study a stochastic aggregation-diffusion equation of the Keller-Segel (KS) type for modeling the chemotaxis in dimensions $d=2,3$. Unlike the classical deterministic KS system, which only allows for idiosyncratic noises, the stochastic KS equation is derived from an interacting particle system subject to both idiosyncratic and common noises. Both the unique existence of solutions to the stochastic KS equation and the mean-field limit result are addressed.
\end{abstract}

Keywords Chemotaxis $\cdot$ Propagation of chaos $\cdot$ Bessel potential $\cdot$ Stochastic partial differential equation

Mathematics Subject Classification 65M75 - 35K55 - 60H30 - 60J7

\section{Introduction}

Many bacteria, such as Escherichia coli, Rhodobacter sphaeroides and Bacillus subtilus, are able to direct their movements according to the surrounding environment

Communicated by Eliot Fried.

The research of J. Qiu is partially supported by the National Science and Engineering Research Council of Canada and the start-up funds from the University of Calgary. H. Huang is partially funded by the DFG research project "Identification of Energies from Observations of Evolutions" (FO767/7-1).

$\bowtie$ Hui Huang

hui.huang@tum.de

Jinniao Qiu

jinniao.qiu@ucalgary.ca

1 Department of Mathematics, Technische Universität München, Boltzmannstr. 3, 85748 Garching, Germany

2 Department of Mathematics and Statistics, University of Calgary, 2500 University Drive NW, Calgary, AB T2N 1N4, Canada 
by a biased random walk. For example, bacteria try to swim toward the highest concentration of nutrition or to flee from poisons. In biology, this phenomenon is called chemotaxis, which describes the directed movement of cells and organisms in response to chemical gradients. Chemotaxis is also observed in other biological fields, for instance the movement of sperm toward the egg during fertilization, the migration of neurons or lymphocytes, and inflammatory processes.

Mathematically, one of the most classical models for studying chemotaxis is the Keller-Segel (KS) equation that was originally proposed in Keller and Segel (1970) to characterize the aggregation of the slime mold amoebae. The classical parabolicelliptic type KS equation is of the following form:

$$
\left\{\begin{array}{l}
\partial_{t} \rho_{t}=\Delta \rho_{t}-\chi \nabla \cdot\left(\rho_{t} \nabla c_{t}\right), \quad x \in \mathbb{R}^{d}, t>0, \\
-\Delta c_{t}=\rho_{t}, \\
\rho_{0} \quad \text { is given, }
\end{array}\right.
$$

where $\rho_{t}(x)$ denotes the bacteria density, and $c_{t}(x)$ represents the chemical substance concentration. The constant $\chi>0$ denotes the chemo-sensitivity or response of the bacteria to the chemical substance. From a mathematical point of view, this equation displays many interesting effects and it has become a topic of intense mathematical research. An important feature of this equation is the competition between the diffusion $\triangle \rho_{t}$ and the nonlocal aggregation $-\chi \nabla \cdot\left(\rho_{t} \nabla c_{t}\right)$. Depending on the choice of the initial mass $m_{0}:=\int_{\mathbb{R}^{d}} \rho_{0}(x) \mathrm{d} x$ and the chemo-sensitivity $\chi$, the solutions to the KS equation may exist globally or blow-up in finite time. In particular, for sufficiently smooth initial conditions, the existence of solutions was verified by Jäger and Luckhaus (1992): if $m_{0} \chi$ is large, then solutions are local in time, and they are global in time if $m_{0} \chi$ is small. For the two-dimensional case, Dolbeault and Perthame (2004) completed the result of Jäger and Luckhaus (1992) by providing an exact value for the critical mass: classical solutions to (1.1) blow-up in finite time when $m_{0} \chi>8 \pi$, and there exists a global in time solution of (1.1) when $m_{0} \chi<8 \pi$. For the case with $m_{0} \chi=8 \pi$, Blanchet et al. (2008) showed that global solutions blow-up in infinite time converging toward a delta diarc distribution at the center of mass. There is an extensive literature on KS systems and their variations, which is out of the scope of this paper. A comprehensive survey on known results related to the KS model from 1970 to 2000 can be found in Horstmann (2003). We also refer to (Hillen and Painter 2009; Perthame 2006; Biler 2018) among many others for more recent developments.

It is also well known that the KS equation (1.1) can be derived from a system of interacting particles $\left\{\left(X_{t}^{i}\right)_{t \geq 0}\right\}_{i=1}^{N}$ satisfying the following form of stochastic differential equations (SDEs):

$$
\mathrm{d} X_{t}^{i}=\frac{\chi}{N-1} \sum_{j \neq i}^{N} F\left(X_{t}^{i}-X_{t}^{j}\right) \mathrm{d} t+\sqrt{2} \mathrm{~d} B_{t}^{i}, \quad i=1, \ldots, N, \quad t>0,
$$

where the process $\left(X_{t}^{i}\right)_{t \geq 0}$ denotes the trajectory of the $i$-th particle, the function $F$ models the pairwise interaction between particles and $\left\{\left(B_{t}^{i}\right)_{t \geq 0}\right\}_{i=1}^{N}$ are $N$ independent 
Wiener processes. The rigorous derivation of the KS equation, for example (1.1), from the microscopic particle system, e.g., (1.2), through the propagation of chaos as $N \rightarrow \infty$ may be found in Huang and Liu (2017a, b), Fournier and Jourdain (2017), Haškovec and Schmeiser (2011), Huang et al. (2019), Fetecau et al. (2019), Bresch et al. (2019). For a review of the topic of the propagation of chaos and the mean-field limit, we refer the readers to Jabin et al. (2017), Carrillo (2014) and the references therein. An asymptotic method, inspired by Hilbert's sixth problem (Hilbert 1902), can also be applied to derive models at the macro-scale (PDEs) from the underlying description at the micro-scale (particle systems); see Bellomo (2016), Burini and Chouhad (2019) for instance.

However, for the classical deterministic KS equation (1.1), the associated particle system (1.2) is only subject to the idiosyncratic noises that are independent from one particle to another, and the effect of the idiosyncratic noises averages out, leading to the deterministic nature of Eq. (1.1). In addition to such idiosyncratic noises, this paper studies the particle systems allowing for common/environmental noises, and the limiting density function satisfies a stochastic partial differential equation of KS type which is new to the best of our knowledge. Common environmental noises (such as temperature, light and sound) are intrinsic to a more realistic setting such as culturing bacteria .

Let $\left(\Omega, \mathcal{F},\left(\mathcal{F}_{t}\right)_{t \geq 0}, \mathbb{P}\right)$ be a complete filtered probability space where the $d^{\prime}$ dimensional Wiener processes $\left\{\left(B_{t}^{i}\right)_{t \geq 0}\right\}_{i=1}^{N}$ are independent of each other as well as of a $d^{\prime}$-dimensional Wiener process $\left(W_{t}\right)_{t \geq 0}{ }^{1}$ The initial data $\zeta^{i}, i=1,2, \ldots, N$ are independently and identically distributed (i.i.d.) with a common density function $\rho_{0}$ and are independent of $\left\{\left(B_{t}^{i}\right)_{t \geq 0}\right\}_{i=1}^{N}$ and $\left(W_{t}\right)_{t \geq 0}$. Denote by $\left(\mathcal{F}_{t}^{W}\right)_{t \geq 0}$ the augmented filtration generated by $\left(W_{t}\right)_{t \geq 0}$.

As the mean-field limit from the interacting particle system that allows for both idiosyncratic and common noises, the stochastic aggregation-diffusion equation of Keller-Segel (KS) type, also called stochastic KS equation, is of the following form:

$$
\left\{\begin{array}{l}
\mathrm{d} \rho_{t}=\frac{1}{2} \sum_{i, j=1}^{d} D_{i j}\left(\rho_{t} \sum_{k=1}^{d^{\prime}}\left(v_{t}^{i k} v_{t}^{j k}+\sigma_{t}^{i k} \sigma_{t}^{j k}\right)\right) \mathrm{d} t-\chi \nabla \cdot\left(\nabla c_{t} \rho_{t}\right) \mathrm{d} t \\
\quad-\sum_{i=1}^{d} D_{i}\left(\rho_{t} \sum_{k=1}^{d^{\prime}} \sigma_{t}^{i k} \mathrm{~d} W_{t}^{k}\right) \\
-\Delta c_{t}+c_{t}=\rho_{t} \\
\rho_{0} \text { is given, }
\end{array}\right.
$$

where $D_{i j}:=\frac{\partial^{2}}{\partial x_{i} \partial x_{j}}, D_{i}:=\frac{\partial}{\partial x_{i}}$, and the leading coefficients $\nu$ and $\sigma$ are deterministic functions from $[0, T] \times \mathbb{R}^{d}$ to $\mathbb{R}^{d \times d^{\prime}}$. One may solve the second equation for the chemical concentration:

$$
c_{t}=(I-\triangle)^{-1} \rho_{t}=\mathcal{G} * \rho_{t}(x),
$$

\footnotetext{
1 The dimension of Wiener process $W$ may be different from $d^{\prime}$; we assume the same dimensionality for notational simplicity.
} 
with $\mathcal{G}$ being the Bessel potential, and it follows that $\nabla c_{t}=\nabla \mathcal{G} * \rho_{t}$ where $\nabla \mathcal{G}$ is called the interaction force. The underlying regularized interacting particle system has the form:

$$
\left\{\begin{array}{l}
\mathrm{d} X_{t}^{i, \varepsilon}=\frac{\chi}{N-1} \sum_{j \neq i}^{N} \nabla \mathcal{G}_{\varepsilon}\left(X_{t}^{i, \varepsilon}-X_{t}^{j, \varepsilon}\right) \mathrm{d} t+v_{t}\left(X_{t}^{i, \varepsilon}\right) \mathrm{d} B_{t}^{i}+\sigma_{t}\left(X_{t}^{i, \varepsilon}\right) \mathrm{d} W_{t}, \quad i=1 \ldots, N, \quad t>0, \\
X_{0}^{i, \varepsilon}=\zeta^{i},
\end{array}\right.
$$

where

$$
\mathcal{G}_{\varepsilon}(x)=\psi_{\varepsilon} * \mathcal{G}(x)=\int_{\mathbb{R}^{d}} \mathcal{G}(y) \psi_{\varepsilon}(x-y) \mathrm{d} y, \quad x \in \mathbb{R}^{d}, \quad \varepsilon>0,
$$

is the regularized Bessel potential with the mollifier function $\psi_{\varepsilon}(x):=\frac{1}{\varepsilon^{d}} \psi\left(\frac{x}{\varepsilon}\right)$ satisfying

$$
0 \leq \psi \in C_{c}^{\infty}\left(\mathbb{R}^{d}\right), \quad \operatorname{supp} \psi \subseteq B(0,1), \quad \int_{B(0,1)} \psi(x) \mathrm{d} x=1
$$

We mention here relevant work (Cattiaux et al. 2016; Fournier and Jourdain 2017) for the existence of solutions to the non-mollified stochastic particle system (1.2). Especially in Fournier and Jourdain (2017), Proposition 4), they proved that for any $N \geq 2$ and $T>0$, if $\left\{\left(X_{t}^{i}\right)_{t \geq 0}\right\}_{i=1}^{N}$ is the solution to (1.2), then

$$
\mathbb{P}\left(\exists s \in[0, T], \exists 1 \leq i<j \leq N: X_{s}^{i}=X_{s}^{j}\right)>0,
$$

i.e., the singularity of the drift term is visited and the particle system is not clearly well-defined. Therefore, in order to obtain a global strong solution to the interacting particle system, we regularize the singular force term $\nabla \mathcal{G}$.

In contrast with the classical KS models (1.1) and (1.2), which only allow for the idiosyncratic noise $\left(B_{t}^{i}\right)_{t \geq 0}$ that is independent from one particle to another, the stochastic systems (1.3) and (1.5) are additionally subject to common noise $\left(W_{t}\right)_{t \geq 0}$, accounting for the common environment where the particles evolve. This common noise leads to the stochastic integrals in stochastic KS equation (1.3), whose (continuous) martingale property and unboundedness result in the inapplicability of classical analysis for deterministic KS equations. In addition, the diffusion coefficients $\sigma$ and $v$ are time-state dependent; along the same lines, a general model may allow for diffusion incorporating Lévy type noises and/or dependence on the density (for instance, see Burini and Chouhad 2019; Escudero 2006; Huang and Liu 2016 for discussions on deterministic KS models with flux limited or fractional diffusion), although we will not seek such a generality herein.

In this paper, we first prove the existence and uniqueness results for both weak and strong solutions to SPDE (1.3). Basically, over a given finite time interval $[0, T]$ when the $L^{4}$-norm of $\rho_{0}$ is sufficiently small, the weak solution exists uniquely and its regularity may be increased for regular initial value $\rho_{0}$ (see Theorems 3.2 and 3.3). 
Then, based on a duality analysis of forward and backward SPDE, we prove that the following stochastic differential equations (SDEs) of McKean-Vlasov type:

$$
\left\{\begin{array}{l}
\mathrm{d} Y_{t}^{i}=\chi \nabla \mathcal{G} * \rho_{t}^{i}\left(Y_{t}^{i}\right) \mathrm{d} t+v\left(Y_{t}^{i}\right) \mathrm{d} B_{t}^{i}+\sigma\left(Y_{t}^{i}\right) \mathrm{d} W_{t}, \quad i=1, \ldots, N, \quad t>0 \\
\rho_{t}^{i} \text { is the conditional density of } Y_{t}^{i} \text { given } \mathcal{F}_{t}^{W} \\
Y_{0}^{i}=\zeta^{i}
\end{array}\right.
$$

has a unique solution with the conditional density $\rho_{t}^{i}$ of $Y_{t}^{i}$ given the common noise $W_{t}$ existing and satisfying SPDE (1.3); see Theorem 4.1. Here by the conditional density $\rho_{t}^{i}$ of $Y_{t}^{i}$ given $\mathcal{F}_{t}^{W}$, we mean that

$$
\mathbb{E}\left[Y_{t}^{i} \in \mathrm{d} x \mid \mathcal{F}_{t}^{W}\right]=\rho_{t}^{i}(x) \mathrm{d} x
$$

, i.e., for any $\varphi \in C_{b}\left(\mathbb{R}^{d}\right)$, it holds that

$$
\mathbb{E}\left[\varphi\left(Y_{t}^{i}\right) \mid \mathcal{F}_{t}^{W}\right]=\int_{\mathbb{R}^{d}} \varphi(x) \rho_{t}^{i}(x) \mathrm{d} x .
$$

Finally, we prove that the solution $\left\{\left(X_{t}^{i, \varepsilon}\right)_{t \geq 0}\right\}_{i=1}^{N}$ of the particle system (1.5) well approximates that of (1.7), which indicates the mean-field limit result, i.e., the empirical measure

$$
\rho_{t}^{\varepsilon, N}:=\frac{1}{N} \sum_{i=1}^{N} \delta_{X_{t}^{i, \varepsilon}}
$$

associated with the particle system (1.5) converges weakly to the unique solution $\rho$ to $\operatorname{SPDE}(1.3)$ as $N \rightarrow \infty$ and $\varepsilon \rightarrow 0^{+}$; see Theorem 5.1 and Corollary 2.

In view of SPDE (1.3) and the particle system (1.5), one may see that when the particle number $N$ tends to infinity, the effect of the idiosyncratic noises averages out, while the effect of common noises does not, leading to the stochastic nature of the limit distribution characterized by SPDE (1.3). We refer to Bensoussan et al. (2013), Carmona et al. (2016), Carmona and Delarue (2018), Coghi and Flandoli (2016) for different models with common noise in the literature. In particular, in a closely related work (Coghi and Flandoli 2016), the authors study the propagation of chaos for an interacting particle system subject to a common environmental noise but with a uniformly Lipschitz continuous potential, and in Choi and Salem (2019), the stochastic mean-field limit of the Cucker-Smale flocking particle system is obtained for a special class of noises. In contrast to the existing literature concerning common noise, the main difficulties in dealing with the proposed stochastic KS models are from the Bessel potential $\mathcal{G}$ which entails the singularity of the drift of SDE (1.7) and the KS type nonlinear and nonlocal properties of SPDE (1.3); in particular, the KS type nonlinear term $-\chi \nabla \cdot\left(\left(\nabla \mathcal{G} * \rho_{t}\right) \rho_{t}\right)$ prevents us from adopting the existing methods in the SPDE literature. Accordingly, the existence and uniqueness of solution 
to SPDE (1.3) is established within sufficiently regular spaces under a divergence-free assumption on coefficient $\sigma$, and we prove that the conditional density exists and satisfies equation (1.3) with a new method based on duality analysis. In addition, for the mean-field limit result, we also introduce regularization with a mollifier function in the particle system (1.5). In this paper, the approaches mix and develop the existing probability theory and stochastic analysis, (S)PDE theory, and the duality analysis in nonlinear filtering theory. Given the outstanding interests shown in the mathematical analysis of biological phenomena, we hope this article will set the stage for further studies on stochastic aggregation-diffusion type equations, opening new perspectives and motivating applied mathematicians to expand the research on this class of models to novel applications.

The rest of the paper is organized as follows. In Sect. 2, we set some notations, present some auxiliary results and give the standing assumptions on the diffusion coefficients. Section 3 is then devoted to the proof of the existence and uniqueness of the weak and strong solution to stochastic KS equation (1.3) in certain regular spaces. On the basis of the well-posedness of SPDE (1.3), we prove the existence and uniqueness of the strong solution to SDE (1.7) in Sect. 4. Finally, the mean-field limit result is addressed in Sect. 5.

\section{Preliminaries}

\subsection{Notations}

The set of all the integers is denoted by $\mathbb{Z}$, with $\mathbb{Z}^{+}$the subset of the strictly positive elements. Denote by $|\cdot|$ (respectively, $\langle\cdot, \cdot\rangle$ or $\cdot$ ) the usual norm (respectively, scalar product) in finite-dimensional Hilbert space such as $\mathbb{R}, \mathbb{R}^{k}, \mathbb{R}^{k \times l}, k, l \in \mathbb{Z}^{+}$. We use $\|f\|_{p}$ for the $L^{p}(1 \leq p \leq \infty)$ norm of a function $f$.

Define the set of multi-indices

$$
\mathcal{A}:=\left\{\alpha=\left(\alpha_{1}, \ldots, \alpha_{d}\right): \alpha_{1}, \ldots, \alpha_{d} \text { are nonnegative integers }\right\}
$$

For any $\alpha \in \mathcal{A}$ and $x=\left(x_{1}, \ldots, x_{d}\right) \in \mathbb{R}^{d}$, denote

$$
|\alpha|=\sum_{i=1}^{d} \alpha_{i}, x^{\alpha}:=x_{1}^{\alpha_{1}} x_{2}^{\alpha_{2}} \ldots x_{d}^{\alpha_{d}}, D^{\alpha}:=\frac{\partial^{|\alpha|}}{\partial x_{1}^{\alpha_{1}} \partial x_{2}^{\alpha_{2}} \ldots \partial x_{d}^{\alpha_{d}}}
$$

For each Banach space $(\mathcal{X},\|\cdot\| \mathcal{X})$, real $q \in[1, \infty]$, and $0 \leq t<\tau \leq T$, we denote by $S_{\mathcal{F}}^{q}([t, \tau] ; \mathcal{X})$ the set of $\mathcal{X}$-valued, $\mathcal{F}_{t \in[0, T] \text {-adapted and continuous pro- }}$ cesses $\left\{X_{s}\right\}_{s \in[t, \tau]}$ such that

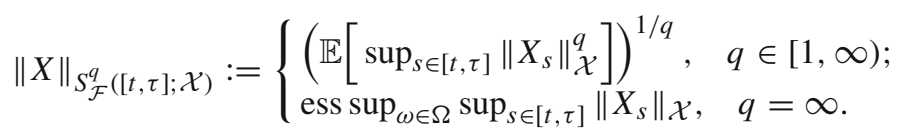


$L_{\mathcal{F}}^{q}(t, \tau ; \mathcal{X})$ denotes the set of (equivalent classes of) $\mathcal{X}$-valued predictable processes $\left\{X_{s}\right\}_{s \in[t, \tau]}$ such that

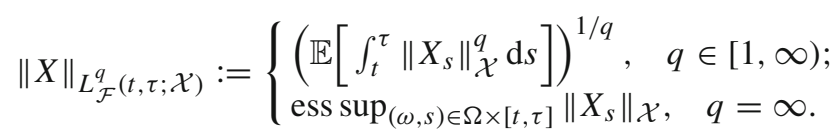

Both $\left(S_{\mathcal{F}}^{q}([t, \tau] ; \mathcal{X}),\|\cdot\|_{S^{q}([t, \tau] \mathcal{X})}\right)$ and $\left(L_{\mathcal{F}}^{q}(t, \tau ; \mathcal{X}),\|\cdot\|_{L_{\mathcal{F}}^{q}(t, \tau ; \mathcal{X})}\right)$ are Banach spaces, and they are well defined with the filtration $\left(\mathcal{F}_{t}\right)_{t \geq 0}$ replaced by $\left(\mathcal{F}_{t}^{W}\right)_{t \geq 0}$.

\subsection{Auxiliary Results and Assumptions}

We first recall some properties of the Bessel potential introduced in (1.4). For $p \in$ $[1, \infty]$, denote by $L^{p}=L^{p}\left(\mathbb{R}^{d}\right)$ the usual Lebesgue integrable spaces with norm $\|\cdot\|_{p}$. Then, for $p \in(1, \infty)$ and $m \in \mathbb{R}$, we may define the space of Bessel potentials (or the Sobolev space with fractional derivatives) (Triebel 1983, p. 37) as

$$
\mathcal{H}_{p}^{m}\left(\mathbb{R}^{d}\right)=\left\{f \mid\|f\|_{\mathcal{H}_{p}^{m}}:=\left\|\mathscr{F}^{-1}\left[\left(1+|\omega|^{2}\right)^{\frac{m}{2}} \mathscr{F}(f)\right]\right\|_{p}<\infty\right\},
$$

where $\mathscr{F}$ is the Fourier transformation. Namely, $\mathcal{H}_{p}^{m}\left(\mathbb{R}^{d}\right)$ (simply written as $\mathcal{H}_{p}^{m}$ ) is defined as space of functions $f$ such that $(1-\triangle)^{\frac{m}{2}} f \in L^{p}\left(\mathbb{R}^{d}\right)$. In (1.4), if $\rho_{t} \in L^{p}$ with $1<p<\infty$, then $c_{t} \in \mathcal{H}_{p}^{2}$. In addition, it holds that

$$
\left\|c_{t}\right\|_{\mathcal{H}_{p}^{2}}=\left\|\mathscr{F}^{-1}\left[\left(1+|\omega|^{2}\right) \mathscr{F}\left[c_{t}\right]\right]\right\|_{p}=\left\|\rho_{t}\right\|_{p}
$$

Due to the equivalence between the Bessel potential space $\mathcal{H}_{p}^{k}\left(\mathbb{R}^{d}\right)$ and the Sobolev space $W^{k, p}\left(\mathbb{R}^{d}\right)(k \in \mathbb{N})$, we have

$$
\left\|\mathcal{G} * \rho_{t}\right\|_{W^{2, p}}=\left\|c_{t}\right\|_{W^{2, p}} \leq C\left\|\rho_{t}\right\|_{p}
$$

Here, the Sobolev space $W^{k, p}\left(\mathbb{R}^{d}\right)$ is defined as

$$
W^{k, p}\left(\mathbb{R}^{d}\right)=\left\{u \in L^{p}\left(\mathbb{R}^{d}\right)\left|D^{\alpha} u \in L^{p}\left(\mathbb{R}^{d}\right), \quad \forall\right| \alpha \mid \leq k\right\}
$$

and

$$
\|u\|_{W^{k, p}}:= \begin{cases}\left(\sum_{|\alpha| \leq k}\left\|D^{\alpha} u\right\|_{L^{p}\left(\mathbb{R}^{d}\right)}^{p}\right)^{\frac{1}{p}} & 1 \leq p<\infty \\ \max _{|\alpha| \leq k}\left\|D^{\alpha} u\right\|_{L^{\infty}\left(\mathbb{R}^{d}\right)} & p=\infty\end{cases}
$$

On the other side, notice that

$$
(I-\triangle)^{-1}=(-\triangle)^{-1}-(-\triangle)^{-1}(I-\triangle)^{-1} .
$$


Thus, we may split the Bessel potential into the Newtonian potential $\Phi$ and a function $\Psi$ such that $\mathscr{F}(\Psi)(\omega)=-\frac{1}{\omega^{2}\left(1+\omega^{2}\right)}$, which implies that $\Psi \in L^{\infty}\left(\mathbb{R}^{d}\right)(d=3)$ or $\nabla \Psi \in L^{\infty}\left(\mathbb{R}^{d}\right)(d=2)$. Namely, one has

$$
\mathcal{G}(x)=\Phi(x)+\Psi(x),
$$

where

$$
\Phi(x)= \begin{cases}\frac{C_{d}}{|x|^{d-2}}, & \text { if } d \geq 3 \\ -\frac{1}{2 \pi} \ln |x|, & \text { if } d=2\end{cases}
$$

is the Newtonian potential. It then follows that for any $\alpha \in \mathcal{A}$ with $|\alpha| \geq 1$, there holds

$$
\begin{aligned}
\left\|D^{\alpha}\left(\nabla \mathcal{G}_{\varepsilon}\right)\right\|_{\infty} & \leq C_{\alpha} \varepsilon^{1-d-|\alpha|}+ \begin{cases}C_{\alpha,\|\Psi\|_{\infty}} \varepsilon^{-1-|\alpha|}, & \text { when } d=3 \\
C_{\alpha,\|\nabla \Psi\|_{\infty}} \varepsilon^{-|\alpha|}, & \text { when } d=2\end{cases} \\
& \leq C_{\alpha} \varepsilon^{1-d-|\alpha|}
\end{aligned}
$$

Here, we have used the estimate $\left\|D^{\alpha}\left(\nabla \Phi_{\varepsilon}\right)\right\|_{\infty} \leq C_{\alpha} \varepsilon^{1-d-|\alpha|}$ from Huang and Liu (2017b, Lemma 2.1).

Following are the standing assumptions on the coefficients $v$ and $\sigma$.

Assumption 1 Given $T>0$ any arbitrary time horizon and $d=2,3$, the measurable diffusion coefficients $\sigma, v:[0, T] \times \mathbb{R}^{d} \longrightarrow \mathbb{R}^{d \times d^{\prime}}$ satisfy

(i) There exists a positive constant $\lambda$ such that

$$
\sum_{i, j=1}^{d} \sum_{k=1}^{d^{\prime}} v_{t}^{i k}(x) v_{t}^{j k}(x) \xi^{i} \xi^{j} \geq \lambda|\xi|^{2}
$$

holds for all $x, \xi \in \mathbb{R}^{d}$ and all $t \geq 0$;

(ii) There exist $m \in \mathbb{Z}^{+}$and real $\Lambda>0$ such that for all $t \in[0, T]$ there holds

$$
\begin{aligned}
& v_{t}^{i k}(\cdot), \sigma_{t}^{i k}(\cdot) \in C^{m}, \text { for } i=1, \ldots, d, k=1, \ldots, d^{\prime}, \\
& \text { and } \sum_{i=1}^{d} \sum_{k=1}^{d^{\prime}}\left\|\sigma_{t}^{i k}(\cdot)\right\|_{C^{m}}+\left\|v_{t}^{i k}(\cdot)\right\|_{C^{m}} \leq \Lambda,
\end{aligned}
$$

where the $C^{m}$ norm is defined as $\|f\|_{C^{m}}=\sum_{|\alpha| \leq m}\left\|D^{\alpha} f\right\|_{\infty}$.

(iii) For all $(t, x) \in[0, T] \times \mathbb{R}^{d}$ and $k=1,2, \ldots, d^{\prime}$,

$$
\sum_{i=1}^{d} D_{i} \sigma_{t}^{i k}(x)=0 .
$$


Remark 2.1 The assumption (i) ensures the superparabolicity of the concerned SPDE, and the boundedness and regularity requirements in (ii) are placed for unique existence of certain regular solutions of SPDE. The readers are referred to Krylov (1999) for more discussions. The divergence-free condition (iii) may be thought of as a technical one for the well-posedness of SPDE (1.3) (see Remark 3.1); on the other hand, the common noise in the stochastic integral term $\sigma_{t}\left(X_{t}^{i, \varepsilon}\right) \mathrm{d} W_{t}$ induces the fluctuations of the velocity field (of the $i$ th particle) formally written as $v_{t}^{i}=\sigma_{t}\left(X_{t}^{i, \varepsilon}\right) \frac{\mathrm{d} W_{t}}{\mathrm{~d} t}$ and in this way, the divergence-free condition means that such fluctuations are of incompressible type. In fact, such kind of divergence-free conditions have been existing in the literature; refer to Brzezniak et al. (2016), Coghi and Flandoli (2016) for more clear and elegant arguments.

In the remaining part of the work, we shall use $C$ to denote a generic constant whose value may vary from line to line, and when needed, a bracket will follow immediately after $C$ to indicate what parameters $C$ depend on. By $A \hookrightarrow B$, we mean that normed space $\left(A,\|\cdot\|_{A}\right)$ is embedded into $\left(B,\|\cdot\|_{B}\right)$ with a constant $C$ such that

$$
\|f\|_{B} \leq C\|f\|_{A}, \quad \forall f \in A .
$$

For readers' convenience, we list Sobolev's embedding theorem in the following lemma, see, e.g., Triebel (1983, p. 129, p. 131) and Brezis (2010, Chapter 9).

Lemma 2.1 There holds the following assertions:

(i) For integer $n>d / q+k$ with $k \in \mathbb{N}$ and $q \in(1, \infty)$, we have $W^{n, q}\left(\mathbb{R}^{d}\right) \hookrightarrow$ $C^{k, \delta}\left(\mathbb{R}^{d}\right)$, for any $\delta \in(0,(n-d / q-k) \wedge 1)$.

(ii) If $1<p_{0}<p_{1}<\infty$ and $-\infty<s_{1}<s_{0}<+\infty$ such that $\frac{d}{p_{0}}-s_{0}=\frac{d}{p_{1}}-s_{1}$, then $\mathcal{H}_{p_{0}}^{s_{0}}\left(\mathbb{R}^{d}\right) \hookrightarrow \mathcal{H}_{p_{1}}^{s_{1}}\left(\mathbb{R}^{d}\right)$ (with Sobolev spaces as special cases ).

\section{Existence and Uniqueness of the Solution to SPDE (1.3)}

This section is devoted to the global existence and uniqueness of the solution to nonlinear SPDE (1.7).

As already noted in (2.1), if $\rho_{t} \in L^{4}$, then it holds that

$$
\left\|c_{t}\right\|_{W^{2,4}}=\left\|\mathcal{G} * \rho_{t}\right\|_{W^{2,4}} \leq S_{d}\left\|\rho_{t}\right\|_{4} .
$$

A direct result of Sobolev's embedding theorem implies

$$
\left\|c_{t}\right\|_{W^{1, \infty}}=\left\|\mathcal{G} * \rho_{t}\right\|_{W^{1, \infty}} \leq\left\|\mathcal{G} * \rho_{t}\right\|_{W^{2,4}} \leq S_{d}\left\|\rho_{t}\right\|_{4},
$$

where $S_{d}$ depends only on $d$.

Before stating the theorem about the well-posedness, we introduce the definition of solutions to $\operatorname{SPDE}(1.3)$. Denote by $C_{c}^{2}\left(\mathbb{R}^{d}\right)$ the space of compactly supported functions having up to second-order continuous derivatives. 
Definition 3.1 A family of random functions $\left\{\rho_{t}(\omega): t \geq 0, \omega \in \Omega\right\}$ lying in $S_{\mathcal{F} W}^{\infty}\left([0, T] ; L^{1} \cap L^{4}\left(\mathbb{R}^{d}\right)\right)$ is a solution to Eq. (1.3) if $\rho_{t}$ satisfies the following stochastic integral equation for all $\varphi \in C_{c}^{2}\left(\mathbb{R}^{d}\right)$,

$$
\begin{aligned}
\left\langle\rho_{t}, \varphi\right\rangle= & \left\langle\rho_{0}, \varphi\right\rangle+\chi \int_{0}^{t}\left\langle\rho_{s}, \nabla \varphi \cdot \nabla c_{s}\right\rangle \mathrm{d} s+\int_{0}^{t}\left\langle\rho_{s}, \sum_{i=1}^{d} D_{i} \varphi \sum_{k=1}^{d^{\prime}} \sigma_{s}^{i k} \mathrm{~d} W_{s}^{k}\right\rangle \\
& +\frac{1}{2} \int_{0}^{t}\left\langle\rho_{s}, \sum_{i, j=1}^{d} D_{i j} \varphi \sum_{k=1}^{d^{\prime}}\left(v_{s}^{i k} \nu_{s}^{j k}+\sigma_{s}^{i k} \sigma_{s}^{j k}\right)\right\rangle \mathrm{d} s .
\end{aligned}
$$

Theorem 3.2 Let Assumption 1 hold with $m=2$. Assume $0 \leq \rho_{0} \in L^{1} \cap \mathcal{H}_{4}^{\frac{1}{2}}\left(\mathbb{R}^{d}\right)^{2}$ with $\left\|\rho_{0}\right\|_{1}=1$. For each $T>0$, there exists a $\kappa>0$ depending only on $T, \chi, \lambda, \Lambda$ and $d$ such that if $\left\|\rho_{0}\right\|_{4} \leq \kappa, \operatorname{SPDE}$ (1.3) admits a unique nonnegative solution in

$$
\begin{aligned}
\mathbb{M}:= & L_{\mathcal{F}^{W}}^{2}\left(0, T ; W^{1,2}\left(\mathbb{R}^{d}\right)\right) \cap L_{\mathcal{F}^{W}}^{4}\left(0, T ; W^{1,4}\left(\mathbb{R}^{d}\right)\right) \\
& \cap S_{\mathcal{F}^{W}}^{\infty}\left([0, T] ; L^{1} \cap L^{4}\left(\mathbb{R}^{d}\right)\right) .
\end{aligned}
$$

Proof The proof is based on delicate estimates of the solution and the latest developments of $L^{p}$-theory of SPDE. First, let

$$
\mathbb{B}:=\left\{u \in S_{\mathcal{F}^{W}}^{\infty}\left([0, T] ; L^{4}\left(\mathbb{R}^{d}\right)\right):\|u\|_{S_{\mathcal{F} W}^{\infty}\left([0, T] ; L^{4}\left(\mathbb{R}^{d}\right)\right)} \leq \ell \kappa\right\}
$$

with metric $d(u, v)=\|u-v\|_{S_{\mathcal{F} W}^{\infty}\left([0, T] ; L^{4}\left(\mathbb{R}^{d}\right)\right)}$, and the positive constants $\kappa$ and $\ell$ are to be determined.

Suppose $\left\|\rho_{0}\right\|_{4} \leq \kappa$. Now, we define a map $\mathcal{T}: \mathbb{B} \rightarrow S_{\mathcal{F}^{W}}^{\infty}\left([0, T] ; L^{4}\left(\mathbb{R}^{d}\right)\right)$ as follows: For each $\xi \in \mathbb{B}$, let $\mathcal{T}(\xi):=\rho^{\xi}$ be the solution to the following linear SPDE:

$$
\left\{\begin{array}{l}
\mathrm{d} \rho_{t}=\left[\frac{1}{2} \sum_{i, j=1}^{d} D_{i j}\left(\rho_{t} \sum_{k=1}^{d^{\prime}}\left(v_{t}^{i k} v_{t}^{j k}+\sigma_{t}^{i k} \sigma_{t}^{j k}\right)\right)-\chi \nabla \cdot\left(\left(\nabla \mathcal{G} * \xi_{t}\right) \rho_{t}\right)\right] \mathrm{d} t \\
\quad-\sum_{i=1}^{d} D_{i}\left(\rho_{t} \sum_{k=1}^{d^{\prime}} \sigma_{t}^{i k} \mathrm{~d} W_{t}^{k}\right) \\
\rho_{0} \text { is given. }
\end{array}\right.
$$

Indeed, as Assumption 1 holds with $m=2$, one may write SPDE (3.5) as a nondivergence form:

$$
\left\{\begin{array}{l}
\mathrm{d} \rho_{t}=\left[\frac{1}{2} \sum_{i, j=1}^{d} \sum_{k=1}^{d^{\prime}}\left(v_{t}^{i k} v_{t}^{j k}+\sigma_{t}^{i k} \sigma_{t}^{j k}\right) D_{i j} \rho_{t} \mathrm{~d} t+F_{t}\left(\rho_{t}\right)\right] \mathrm{d} t \\
\quad-\sum_{i=1}^{d} \sum_{k=1}^{d^{\prime}} \sigma_{t}^{i k} D_{i} \rho_{t} \mathrm{~d} W_{t}^{k} \\
\rho_{0} \text { is given, }
\end{array}\right.
$$

\footnotetext{
$\overline{2}$ Here, the initial condition $\rho_{0} \in \mathcal{H}_{4}^{\frac{1}{2}}\left(\mathbb{R}^{d}\right)$ is required by the $L^{p}$-theory of SPDEs (see Krylov 1999, Theorem 5.1) for $p=4$. 
with

$$
\begin{aligned}
F_{t}\left(\rho_{t}\right):= & \frac{1}{2} \sum_{i, j=1}^{d} D_{i}\left(\rho_{t} \sum_{k=1}^{d^{\prime}} D_{j}\left(v_{t}^{i k} v_{t}^{j k}+\sigma_{t}^{i k} \sigma_{t}^{j k}\right)\right) \\
& +\frac{1}{2} \sum_{i, j=1}^{d} D_{j} \rho_{t} \sum_{k=1}^{d^{\prime}} D_{i}\left(v_{t}^{i k} v_{t}^{j k}+\sigma_{t}^{i k} \sigma_{t}^{j k}\right) \\
& -\chi \nabla \cdot\left(\left(\nabla \mathcal{G} * \xi_{t}\right) \rho_{t}\right),
\end{aligned}
$$

where we have used Assumption 1 (iii) for the stochastic integral, i.e.,

$$
\sum_{j=1}^{d} D_{j}\left(\sigma_{s}^{j k} \rho_{s}\right)=\sum_{j=1}^{d} \rho_{s} D_{j} \sigma_{s}^{j k}+\sum_{j=1}^{d} D_{j} \rho_{s} \sigma_{s}^{j k}=\sum_{j=1}^{d} D_{j} \rho_{s} \sigma_{s}^{j k}
$$

For each $\xi \in \mathbb{B}$ and $\rho_{t} \in L^{p}\left(\mathbb{R}^{d}\right)$ with $p \in\{2,4\}$, relation (3.2) indicates that

$$
\left\|\left(\nabla \mathcal{G} * \xi_{t}\right) \rho_{t}\right\|_{p} \leq\left\|\nabla \mathcal{G} * \xi_{t}\right\|_{\infty}\left\|\rho_{t}\right\|_{p} \leq S_{d}\left\|\xi_{t}\right\|_{4}\left\|\rho_{t}\right\|_{p} \leq \ell S_{d} \kappa\left\|\rho_{t}\right\|_{p}, \quad \text { a.s., for all } t \in[0, T] .
$$

This together with Assumption 1 allows us, through standard computations, to check that the conditions of the $L^{p}$-theory of SPDE (see Krylov 1999, Theorems 5.1 and 7.1 for the case when $n=-1$ therein) and the maximum principle (Krylov 1999, Theorem 5.12) are satisfied and we conclude that the linear $\operatorname{SPDE}(3.5)$ admits a unique solution $\rho^{\xi}$ which is nonnegative and lying in $L_{\mathcal{F}^{W}}^{p}\left(0, T ; W^{1, p}\left(\mathbb{R}^{d}\right)\right) \cap S_{\mathcal{F}^{W}}^{p}\left([0, T] ; L^{p}\left(\mathbb{R}^{d}\right)\right)$, $p \in\{2,4\}$.

Next, we check that $\rho^{\xi} \in S_{\mathcal{F} W}^{\infty}\left([0, T] ; L^{1} \cap L^{4}\left(\mathbb{R}^{d}\right)\right)$ and without causing confusion we drop the superscript $\xi$. It is easy to see that the solution of (3.5) has the property of conservation of mass, i.e.,

$$
\left\|\rho_{t}\right\|_{1}=\left\|\rho_{0}\right\|_{1}=1 \quad \text { a.s.. }
$$

Applying the Itô formula for $L^{p}$-norms in Krylov (2010, Theorem 2.1), we have for any $0<t \leq T$

$$
\begin{aligned}
& \left\|\rho_{t}\right\|_{4}^{4}-\left\|\rho_{0}\right\|_{4}^{4} \\
& =\int_{0}^{t}\left(\sum_{i, j=1}^{d} \sum_{k=1}^{d^{\prime}}-\left\langle 6\left|\rho_{s}\right|^{2} D_{i} \rho_{s}, D_{j}\left(\left(v_{s}^{i k} v_{s}^{j k}+\sigma_{s}^{i k} \sigma_{s}^{j k}\right) \rho_{s}\right)\right\rangle\right. \\
& +6 \sum_{k=1}^{d^{\prime}}\left\langle\left|\rho_{s}\right|^{2},\left|\sum_{j=1}^{d} D_{j}\left(\rho_{s} \sigma_{s}^{j k}\right)\right|^{2}\right\rangle \\
& \left.\quad+12 \chi\left\langle\rho_{s}\left(\nabla \rho_{s}\right),\left(\nabla \mathcal{G} * \xi_{s}\right) \rho_{s}^{2}\right\rangle\right) \mathrm{d} s+12 \sum_{i=1}^{d} \sum_{k=1}^{d^{\prime}} \int_{0}^{t}\left\langle\left|\rho_{s}\right|^{2} D_{i} \rho_{s}, \sigma_{s}^{i k} \rho_{s}\right\rangle \mathrm{d} W_{s}^{k} \text { a.s.. }
\end{aligned}
$$


Due to (iii) in Assumption 1, we know that for $k=1,2, \ldots, d^{\prime}$,

$12 \sum_{i=1}^{d}\left\langle\left|\rho_{s}\right|^{2} D_{i} \rho_{s}, \sigma^{i k} \rho_{s}\right\rangle=3 \sum_{i=1}^{d}\left\langle D_{i}\left(\left|\rho_{s}\right|^{4}\right), \sigma_{s}^{i k}\right\rangle=-3\left\langle\left|\rho_{s}\right|^{4}, \sum_{i=1}^{d} D_{i} \sigma_{s}^{i k}\right\rangle=0$.

Thus, one has

$$
\begin{aligned}
\left\|\rho_{t}\right\|_{4}^{4} & -\left\|\rho_{0}\right\|_{4}^{4} \\
= & -\int_{0}^{t} \sum_{i, j=1}^{d} \sum_{k=1}^{d^{\prime}}\left\langle 6\left|\rho_{s}\right|^{2} D_{i} \rho_{s},\left(v_{s}^{i k} v_{s}^{j k}+\sigma_{s}^{i k} \sigma_{s}^{j k}\right) D_{j} \rho_{s}\right\rangle \mathrm{d} s \\
& -\sum_{i, j=1}^{d} \sum_{k=1}^{d^{\prime}} \int_{0}^{t}\left\langle 6\left|\rho_{s}\right|^{2} D_{i} \rho_{s}, D_{j}\left(v_{s}^{i k} v_{s}^{j k}+\sigma_{s}^{i k} \sigma_{s}^{j k}\right) \rho_{s}\right\rangle \mathrm{d} s \\
& +\int_{0}^{t} 6 \sum_{k=1}^{d^{\prime}}\left\langle\left|\rho_{s}\right|^{2},\left|\sum_{j=1}^{d} D_{j}\left(\rho_{s} \sigma_{s}^{j k}\right)\right|^{2}\right\rangle+12 \chi\left\langle\rho_{s}\left(\nabla \rho_{s}\right),\left(\nabla \mathcal{G} * \xi_{s}\right) \rho_{s}^{2}\right\rangle \mathrm{d} s \quad \text { a.s.. }
\end{aligned}
$$

Using (iii) in Assumption 1 as in (3.7) again yields that

$$
\begin{aligned}
& -\sum_{i, j=1}^{d} \sum_{k=1}^{d^{\prime}}\left\langle 6\left|\rho_{s}\right|^{2} D_{i} \rho_{s},\left(v_{s}^{i k} v_{s}^{j k}+\sigma_{s}^{i k} \sigma_{s}^{j k}\right) D_{j} \rho_{s}\right\rangle \\
& =-\sum_{i, j=1}^{d} \sum_{k=1}^{d^{\prime}}\left\langle 6\left|\rho_{s}\right|^{2} D_{i} \rho_{s},\left(v_{s}^{i k} v_{s}^{j k}\right) D_{j} \rho_{s}\right\rangle-\sum_{k=1}^{d^{\prime}}\left\langle 6\left|\rho_{s}\right|^{2},\left|\sum_{j=1}^{d} D_{j} \rho_{s} \sigma_{s}^{j k}\right|^{2}\right\rangle \\
& =-\sum_{i, j=1}^{d} \sum_{k=1}^{d^{\prime}}\left\langle 6\left|\rho_{s}\right|^{2} D_{i} \rho_{s},\left(v_{s}^{i k} v_{s}^{j k}\right) D_{j} \rho_{s}\right\rangle-\sum_{k=1}^{d^{\prime}}\left\langle 6\left|\rho_{s}\right|^{2},\left|\sum_{j=1}^{d} D_{j}\left(\rho_{s} \sigma_{s}^{j k}\right)\right|^{2}\right\rangle .
\end{aligned}
$$

Therefore, it holds that

$$
\begin{aligned}
\left\|\rho_{t}\right\|_{4}^{4}-\left\|\rho_{0}\right\|_{4}^{4} & \\
= & -\int_{0}^{t} \sum_{i, j=1}^{d} \sum_{k=1}^{d^{\prime}}\left\langle 6\left|\rho_{s}\right|^{2} D_{i} \rho_{s},\left(v_{s}^{i k} v_{s}^{j k}\right) D_{j} \rho_{s}\right\rangle \mathrm{d} s \\
& -\int_{0}^{t} \sum_{i, j=1}^{d} \sum_{k=1}^{d^{\prime}}\left\langle 6\left|\rho_{s}\right|^{2} D_{i} \rho_{s}, D_{j}\left(v_{s}^{i k} v_{s}^{j k}+\sigma_{s}^{i k} \sigma_{s}^{j k}\right) \rho_{s}\right\rangle \mathrm{d} s \\
& +12 \chi \int_{0}^{t}\left\langle\rho_{s}\left(\nabla \rho_{s}\right),\left(\nabla \mathcal{G} * \xi_{s}\right) \rho_{s}^{2}\right\rangle \mathrm{d} s, \quad \text { a.s.. }
\end{aligned}
$$


It follows from (i) in Assumption 1 that

$$
-\sum_{i, j=1}^{d} \sum_{k=1}^{d^{\prime}}\left\langle 6\left|\rho_{s}\right|^{2} D_{i} \rho_{s},\left(v_{s}^{i k} v_{s}^{j k}\right) D_{j} \rho_{s}\right\rangle \leq-6 \lambda\left\|\rho_{s} \nabla \rho_{s}\right\|_{2}^{2},
$$

and by (ii) in Assumption 1 one has

$$
\begin{aligned}
& -6 \sum_{i, j=1}^{d} \sum_{k=1}^{d^{\prime}}\left\langle\left|\rho_{s}\right|^{2} D_{i} \rho_{s}, D_{j}\left(v_{s}^{i k} v_{s}^{j k}+\sigma_{s}^{i k} \sigma_{s}^{j k}\right) \rho_{s}\right\rangle \\
& \leq 24 \Lambda^{2}\left\langle\left|\rho_{s}\right|^{2}\left|\nabla \rho_{s}\right|, \rho_{s}\right\rangle \\
& \leq 2 \lambda\left\|\rho_{s} \nabla \rho_{s}\right\|_{2}^{2}+\frac{\left(12 \Lambda^{2}\right)^{2}}{2 \lambda}\left\|\rho_{s}\right\|_{4}^{4} .
\end{aligned}
$$

We also notice that

$$
\begin{aligned}
12 \chi\left\langle\rho_{s}\left(\nabla \rho_{s}\right),\left(\nabla \mathcal{G} * \xi_{s}\right) \rho_{s}^{2}\right\rangle & \leq 12 \chi\left\|\rho_{s} \nabla \rho_{s}\right\|_{2}\left\|\rho_{s}\right\|_{4}^{2}\left\|\nabla \mathcal{G} * \xi_{s}\right\|_{\infty} \\
(\text { by relation(3.2)) } & \leq 12 \chi S_{d}\left\|\rho_{s} \nabla \rho_{s}\right\| 2\left\|\rho_{s}\right\|_{4}^{2}\left\|\xi_{s}\right\|_{4} \leq 12 \ell \chi S_{d} \kappa\left\|\rho_{s} \nabla \rho_{s}\right\| 2\left\|\rho_{s}\right\|_{4}^{2} \\
\text { (by Young's inequality) } & \leq 2 \lambda\left\|\rho_{s} \nabla \rho_{s}\right\|_{2}^{2}+\frac{\left(6 \ell \chi S_{d} \kappa\right)^{2}}{2 \lambda}\left\|\rho_{s}\right\|_{4}^{4} .
\end{aligned}
$$

Collecting above estimates, (3.9) yields that

$$
\begin{aligned}
& \left\|\rho_{t}\right\|_{4}^{4}-\left\|\rho_{0}\right\|_{4}^{4} \\
& \quad \leq-6 \lambda \int_{0}^{t}\left\|\rho_{s} \nabla \rho_{s}\right\|_{2}^{2} \mathrm{~d} s+4 \lambda \int_{0}^{t}\left\|\rho_{s} \nabla \rho_{s}\right\|_{2}^{2} \mathrm{~d} s+\left(\frac{\left(12 \Lambda^{2}\right)^{2}}{2 \lambda}+\frac{\left(6 \ell \chi S_{d} \kappa\right)^{2}}{2 \lambda}\right) \int_{0}^{t}\left\|\rho_{s}\right\|_{4}^{4} \mathrm{~d} s \\
& \quad \leq\left(\frac{\left(12 \Lambda^{2}\right)^{2}}{2 \lambda}+\frac{\left(6 \ell \chi S_{d} \kappa\right)^{2}}{\lambda}\right) \int_{0}^{t}\left\|\rho_{s}\right\|_{4}^{4} \mathrm{~d} s .
\end{aligned}
$$

Take a sufficiently large $\ell>1$ and relatively small $\kappa_{0}{ }^{3}$ such that whenever $\kappa \leq \kappa_{0}$ it holds that

$$
\exp \left\{\frac{T}{4}\left(\frac{\left(12 \Lambda^{2}\right)^{2}}{2 \lambda}+\frac{\left(6 \ell \chi S_{d} \kappa\right)^{2}}{\lambda}\right)\right\} \leq \ell
$$

3 The selections of $\ell$ and $\kappa$ are not unique; a particular case is to take $\kappa_{0} \leq \frac{1}{\chi \ell}$ with

$$
\ell=\exp \left\{\frac{T}{4}\left(\frac{\left(12 \Lambda^{2}\right)^{2}}{2 \lambda}+\frac{\left(6 S_{d}\right)^{2}}{\lambda}\right)\right\}
$$


Applying Gronwall's inequality to (3.12) yields that

$$
\begin{aligned}
\sup _{t \in[0, T]}\left\|\rho_{t}\right\|_{4} & \leq\left\|\rho_{0}\right\|_{4} \exp \left\{\frac{T}{4}\left(\frac{\left(12 \Lambda^{2}\right)^{2}}{2 \lambda}+\frac{\left(6 \ell \chi S_{d} \kappa\right)^{2}}{\lambda}\right)\right\}, \\
& \leq \ell \kappa,
\end{aligned}
$$

which gives that $\rho \in \mathbb{B}$.

Fix the constants $\ell$ and $\kappa_{0}$ as selected above. Let $\kappa \leq \kappa_{0}$. For all $\xi \in \mathbb{B}$, let $\rho^{\xi}$ be the unique solution of the linear SPDE (3.5). From the discussion above, we get the solution map

$$
\mathcal{T}: \quad \mathbb{B} \rightarrow \mathbb{B}, \quad \xi \mapsto \rho^{\xi}
$$

Next, we show that the map $\mathcal{T}$ is a contraction.

For any $\bar{\xi}, \hat{\xi} \in \mathbb{B}$, set $\delta \rho=\rho^{\bar{\xi}}-\rho^{\hat{\xi}}$ and $\delta \xi=\bar{\xi}-\hat{\xi}$. As before, we apply Itô formula for the $L^{4}$-norm of $\delta \rho$ :

$$
\begin{aligned}
& \left\|\delta \rho_{t}\right\|_{4}^{4} \\
& =\int_{0}^{t}\left(-\sum_{i, j=1}^{d} \sum_{k=1}^{d^{\prime}}\left\langle 6\left|\delta \rho_{s}\right|^{2} D_{i} \delta \rho_{s}, D_{j}\left(\left(v_{s}^{i k} v_{s}^{j k}+\sigma_{s}^{i k} \sigma_{s}^{j k}\right) \delta \rho_{s}\right)\right\rangle\right. \\
& \left.+6 \sum_{k=1}^{d^{\prime}}\left\langle\left|\delta \rho_{s}\right|^{2},\left|\sum_{j} D_{j}\left(\delta \rho_{s} \sigma_{s}^{j k}\right)\right|^{2}\right\rangle\right) \mathrm{d} s \\
& +\int_{0}^{t} 12 \chi\left\langle\left|\delta \rho_{s}\right|^{2} \nabla \delta \rho_{s}, \nabla \mathcal{G} * \bar{\xi}_{s} \rho_{s}^{\bar{\xi}}-\nabla \mathcal{G} * \hat{\xi}_{s} \rho_{s}^{\hat{\xi}}\right\rangle \mathrm{d} s \\
& +12 \sum_{i=1}^{d} \sum_{k=1}^{d^{\prime}} \int_{0}^{t}\left\langle\left|\delta \rho_{s}\right|^{2} D_{i} \delta \rho_{s}, \sigma_{s}^{i k} \delta \rho_{s}\right\rangle \mathrm{d} W_{s}^{k} \\
& =\int_{0}^{t}\left(-\sum_{i, j=1}^{d} \sum_{k=1}^{d^{\prime}}\left\langle 6\left|\delta \rho_{s}\right|^{2} D_{i} \delta \rho_{s},\left(v_{s}^{i k} v_{s}^{j k}\right) D_{j} \delta \rho_{s}\right\rangle\right. \\
& \left.-\sum_{i, j=1}^{d} \sum_{k=1}^{d^{\prime}}\left\langle 6\left|\delta \rho_{s}\right|^{2} D_{i} \delta \rho_{s}, D_{j}\left(v_{s}^{i k} v_{s}^{j k}+\sigma_{s}^{i k} \sigma_{s}^{j k}\right) \delta \rho_{s}\right\rangle\right) \mathrm{d} s \\
& +\int_{0}^{t} 12 \chi\left\langle\left|\delta \rho_{s}\right|^{2} \nabla \delta \rho_{s}, \nabla \mathcal{G} * \bar{\xi}_{s} \rho_{s}^{\bar{\xi}}-\nabla \mathcal{G} * \hat{\xi}_{s} \rho_{s}^{\hat{\xi}}\right\rangle \mathrm{d} s \\
& \leq-6 \lambda \int_{0}^{t}\left\|\delta \rho_{s} \nabla \delta \rho_{s}\right\|_{2}^{2} \mathrm{~d} s+2 \lambda \int_{0}^{t}\left\|\delta \rho_{s} \nabla \delta \rho_{s}\right\|_{2}^{2} d s+\frac{\left(12 \Lambda^{2}\right)^{2}}{2 \lambda} \int_{0}^{t}\left\|\delta \rho_{s}\right\|_{4}^{4} \mathrm{~d} s \\
& +\int_{0}^{t} 12 \chi\left\langle\left|\delta \rho_{s}\right|^{2} \nabla \delta \rho_{s}, \nabla \mathcal{G} * \bar{\xi}_{s} \rho_{s}^{\bar{\xi}}-\nabla \mathcal{G} * \hat{\xi}_{s} \rho_{s}^{\hat{\xi}}\right\rangle \mathrm{d} s, \quad \text { a.s.. }
\end{aligned}
$$


Let us compute that

$$
\begin{aligned}
12 \chi & \left\langle\left|\delta \rho_{s}\right|^{2} \nabla \delta \rho_{s}, \nabla \mathcal{G} * \delta \xi_{s} \rho_{s}^{\bar{\xi}}\right\rangle \\
& \leq 12 \chi\left\|\delta \rho_{s} \nabla \delta \rho_{s}\right\|_{2}\left\|\delta \rho_{s} \rho_{s}^{\bar{\xi}}\right\|_{2}\left\|\nabla \mathcal{G} * \delta \xi_{s}\right\|_{\infty} \\
& \leq 12 \chi S_{d}\left\|\delta \rho_{s} \nabla \delta \rho_{s}\right\|_{2}\left\|\delta \rho_{s}\right\|_{4}\left\|\rho_{s}^{\bar{\xi}}\right\|_{4}\left\|\delta \xi_{s}\right\|_{4} \\
& \leq 2 \lambda\left\|\delta \rho_{s} \nabla \delta \rho_{s}\right\|_{2}^{2}+\frac{\left(6 \chi S_{d}\right)^{2}}{2 \lambda}\left\|\delta \rho_{s}\right\|_{4}^{2}\left\|\rho_{s}^{\bar{\xi}}\right\|_{4}^{2}\left\|\delta \xi_{s}\right\|_{4}^{2} \\
& \leq 2 \lambda\left\|\delta \rho_{s} \nabla \delta \rho_{s}\right\|_{2}^{2}+\frac{\left(6 \chi S_{d}\right)^{2}}{4 \lambda}\left((\ell \kappa)^{2}\left\|\delta \rho_{s}\right\|_{4}^{4}+(\ell \kappa)^{-2}\left\|\rho_{s}^{\bar{\xi}}\right\|_{4}^{4}\left\|\delta \xi_{s}\right\|_{4}^{4}\right) \\
& \leq 2 \lambda\left\|\delta \rho_{s} \nabla \delta \rho_{s}\right\|_{2}^{2}+\frac{\left(6 \ell \chi S_{d} \kappa\right)^{2}}{4 \lambda}\left\|\delta \rho_{s}\right\|_{4}^{4}+\frac{\left(6 \ell \chi S_{d} \kappa\right)^{2}}{4 \lambda}\left\|\delta \xi_{s}\right\|_{4}^{4} .
\end{aligned}
$$

In a similar way to (3.11), we have

$$
12 \chi\left\langle\left|\delta \rho_{s}\right|^{2} \nabla \delta \rho_{s}, \nabla \mathcal{G} * \hat{\xi}_{s} \delta \rho_{s}\right\rangle \leq 2 \lambda\left\|\delta \rho_{s} \nabla \delta \rho_{s}\right\|_{2}^{2}+\frac{\left(6 \ell \chi S_{d} \kappa\right)^{2}}{2 \lambda}\left\|\delta \rho_{s}\right\|_{4}^{4} .
$$

Thus, combining above estimates gives

$$
\begin{aligned}
& 12 \chi\left\langle\left|\delta \rho_{s}\right|^{2} \nabla \delta \rho_{s}, \nabla \mathcal{G} * \bar{\xi}_{s} \rho_{s}^{\bar{\xi}}-\nabla \mathcal{G} * \hat{\xi}_{s} \rho_{s}^{\hat{\xi}}\right\rangle=12 \chi\left\langle\left|\delta \rho_{s}\right|^{2} \nabla \delta \rho_{s}, \nabla \mathcal{G} * \delta \xi_{s} \rho_{s}^{\bar{\xi}}+\nabla \mathcal{G} * \hat{\xi}_{s} \delta \rho_{s}\right\rangle \\
& \leq 4 \lambda\left\|\delta \rho_{s} \nabla \delta \rho_{s}\right\|_{2}^{2}+\frac{\left(6 \ell \chi S_{d} \kappa\right)^{2}}{\lambda}\left\|\delta \rho_{s}\right\|_{4}^{4}+\frac{\left(6 \ell \chi S_{d} \kappa\right)^{2}}{4 \lambda}\left\|\delta \xi_{s}\right\|_{4}^{4},
\end{aligned}
$$

which together with (3.14) and (3.13) implies

$$
\begin{aligned}
\left\|\delta \rho_{t}\right\|_{4}^{4} & \leq\left(\frac{\left(12 \Lambda^{2}\right)^{2}}{2 \lambda}+\frac{\left(6 \ell \chi S_{d} \kappa\right)^{2}}{\lambda}\right) \int_{0}^{t}\left\|\delta \rho_{s}\right\|_{4}^{4} \mathrm{~d} s+\frac{\left(6 \ell \chi S_{d} \kappa\right)^{2}}{4 \lambda} \int_{0}^{t}\left\|\delta \xi_{s}\right\|_{4}^{4} \mathrm{~d} s \\
& \leq \frac{4 \ln \ell}{T} \int_{0}^{t}\left\|\delta \rho_{s}\right\|_{4}^{4} \mathrm{~d} s+\frac{\left(6 \ell \chi S_{d} \kappa\right)^{2}}{4 \lambda} \int_{0}^{t}\left\|\delta \xi_{s}\right\|_{4}^{4} \mathrm{~d} s \quad \text { a.s.. }
\end{aligned}
$$

By Gronwall's inequality, we get

$$
\|\delta \rho\|_{S_{\mathcal{F} W}^{\infty}\left([0, T] ; L^{4}\left(\mathbb{R}^{d}\right)\right)} \leq\left[\frac{\left(6 \ell \chi S_{d} \kappa\right)^{2} T}{4 \lambda}\right]^{\frac{1}{4}} \ell\|\delta \xi\|_{S_{\mathcal{F} W}^{\infty}\left([0, T] ; L^{4}\left(\mathbb{R}^{d}\right)\right)} \quad \text { a.s.. }
$$

Hence, whenever $0<\kappa<\kappa_{0} \wedge\left[\frac{4 \lambda}{\left(6 \ell^{3} \chi S_{d}\right)^{2} T}\right]^{\frac{1}{2}}$, the solution map $\mathcal{T}$ is a contraction mapping on the complete metric space $\mathbb{B}$, and it admits a unique fixed point $\rho=\rho^{\rho}$ which is the unique solution to SPDE (1.3).

Remark 3.1 For the well-posedness of SPDE (1.3), the main difficulty lies in the KS type nonlinear term $-\chi \nabla \cdot\left(\left(\nabla \mathcal{G} * \rho_{t}\right) \rho_{t}\right)$ which prevents us from using the existing methods in the SPDE literature. In view of Eq. (3.8) and the computation that follows, one may see that the stochastic integral there equals zero because of the 
divergence-free condition (iii) of Assumption 1. This further allows us to obtain $\rho \in S_{\mathcal{F}^{W}}^{\infty}\left(0, T ; L^{4}\left(\mathbb{R}^{d}\right)\right)$ which finally yields the conclusions in Theorem 3.2 with a deterministic $\kappa$. Without (iii) of Assumption 1, one may try to generalize the localization technique with stopping times (see Karatzas and Shreve 1998, Chapter 1, Section 5) for random fields which, however, may incur cumbersome arguments not just for the well-posedness of SPDE (1.3) in this section, but also for the subsequent sections.

In view of the above proof, we can particularly take

$$
0<\chi \kappa<\frac{1}{\ell} \wedge\left[\frac{4 \lambda}{\left(6 \ell^{3} S_{d}\right)^{2} T}\right]^{\frac{1}{2}}, \quad \text { with } \ell=\exp \left\{\frac{T}{4}\left(\frac{\left(12 \Lambda^{2}\right)^{2}}{2 \lambda}+\frac{\left(6 S_{d}\right)^{2}}{\lambda}\right)\right\}
$$

for the well-posedness of SPDE (1.3) in Theorem 3.2. Therefore, whenever $\chi\left\|\rho_{0}\right\|_{4}<$ $\frac{1}{\ell} \wedge\left[\frac{4 \lambda}{\left(6 \ell^{3} S_{d}\right)^{2} T}\right]^{\frac{1}{2}}$, the unique existence of solution in $\mathbb{M}$ can be asserted as in Theorem 3.2 . i.e.,

Furthermore, suppose that the diffusion coefficients $v$ and $\sigma$ are spatial invariant, the measurable diffusion coefficients $\sigma, v:\left([0, T], \mathcal{B}([0, T]) \longrightarrow\left(\mathbb{R}^{d \times d^{\prime}}, \mathcal{B}\left(\mathbb{R}^{d \times d^{\prime}}\right)\right)\right.$.

Then, the left-hand side of (3.10) and the third term of line (3.14) will vanish. Repeating the proof and combining computations around (3.13) and (3.16), we can obtain the well-posedness of SPDE (1.3) in Theorem 3.2 with a particular selection:

$$
0<\chi \kappa \sqrt{T}<\frac{1}{\ell} \wedge\left[\frac{4 \lambda}{\left(6 \ell^{3} S_{d}\right)^{2}}\right]^{\frac{1}{2}}, \quad \text { with } \ell=\exp \left\{\frac{\left(6 S_{d}\right)^{2}}{4 \lambda}\right\}
$$

which indicates that for any given $\rho_{0}$, the existence and uniqueness of solution may be guaranteed on time interval $\left[0, T_{0}\right]$ if

$$
T_{0}<\frac{1}{\ell^{2} \chi^{2}\left\|\rho_{0}\right\|_{4}^{2}} \wedge \frac{4 \lambda}{\left(6 \ell^{3} \chi\left\|\rho_{0}\right\|_{4} S_{d}\right)^{2}}
$$

For this solution on $\left[0, T_{0}\right]$, we may conduct estimates as in the proof of Theorem 3.2. Notice that instead of (3.11) and (3.12), we have

$$
\begin{aligned}
12 \chi\left\langle\rho_{s}\left(\nabla \rho_{s}\right),\left(\nabla \mathcal{G} * \rho_{s}\right) \rho_{s}^{2}\right\rangle & \leq 12 \chi\left\|\rho_{s} \nabla \rho_{s}\right\|_{2}\left\|\rho_{s}\right\|_{4}^{2}\left\|\nabla \mathcal{G} * \rho_{s}\right\|_{\infty} \\
& \leq 12 \chi S_{d}\left\|\rho_{s} \nabla \rho_{s}\right\| 2\left\|\rho_{s}\right\|_{4}^{2}\left\|\rho_{s}\right\|_{4} \\
& \leq 2 \lambda\left\|\rho_{s} \nabla \rho_{s}\right\|_{2}^{2}+\frac{\left(6 \chi S_{d}\right)^{2}}{2 \lambda}\left\|\rho_{s}\right\|_{4}^{6},
\end{aligned}
$$


and

$$
\begin{aligned}
& \left\|\rho_{t}\right\|_{4}^{4}-\left\|\rho_{0}\right\|_{4}^{4} \\
& \quad \leq-6 \lambda \int_{0}^{t}\left\|\rho_{s} \nabla \rho_{s}\right\|_{2}^{2} \mathrm{~d} s+2 \lambda \int_{0}^{t}\left\|\rho_{s} \nabla \rho_{s}\right\|_{2}^{2} d s+\frac{\left(6 \chi S_{d}\right)^{2}}{2 \lambda} \int_{0}^{t}\left\|\rho_{s}\right\|_{4}^{4} \mathrm{~d} s \\
& \quad \leq-\lambda \int_{0}^{t}\left\|\nabla\left(\rho_{s}^{2}\right)\right\|_{2}^{2} \mathrm{~d} s+\frac{\left(6 \chi S_{d}\right)^{2}}{2 \lambda} \int_{0}^{t}\left\|\rho_{s}\right\|_{4}^{6} \mathrm{~d} s .
\end{aligned}
$$

Meanwhile, using the Gagliardo-Nirenberg inequality yields that there exists a constant $N_{d}>0$ depending on $d$ such that

$$
\begin{aligned}
\left\|\rho_{s}\right\|_{4}^{2}=\left\|\rho_{s}^{2}\right\|_{2} \leq N_{d}\left\|\nabla\left(\rho_{s}^{2}\right)\right\|_{2}^{\frac{d}{d+2}} \cdot\left\|\rho_{s}^{2}\right\|_{1}^{\frac{2}{d+2}} & =N_{d}\left\|\nabla\left(\rho_{s}^{2}\right)\right\|_{2}^{\frac{d}{d+2}} \cdot\left\|\rho_{s}\right\|_{2}^{\frac{4}{d+2}} \\
\text { (by interpolation inequality) } & \leq N_{d}\left\|\nabla\left(\rho_{s}^{2}\right)\right\|_{2}^{\frac{d}{d+2}} \cdot\left\|\rho_{s}\right\|_{4}^{\frac{8}{3(d+2)}} \cdot\left\|\rho_{s}\right\|_{1}^{\frac{4}{3(d+2)}} \\
& =N_{d}\left\|\nabla\left(\rho_{s}^{2}\right)\right\|_{2}^{\frac{d}{d+2}} \cdot\left\|\rho_{s}\right\|_{4}^{\frac{8}{3(d+2)}} .
\end{aligned}
$$

Then, it follows that

$$
\left\|\nabla\left(\rho_{s}^{2}\right)\right\|_{2}^{2} \geq\left|N_{d}\right|^{-\frac{2(d+2)}{d}} \cdot\left\|\rho_{s}\right\|_{4}^{4+\frac{8}{3 d}},
$$

which inserted into (3.19) gives

$$
\left\|\rho_{t}\right\|_{4}^{4}-\left\|\rho_{0}\right\|_{4}^{4} \leq \int_{0}^{t}\left\|\rho_{s}\right\|_{4}^{4+\frac{8}{3 d}}\left(\frac{\left(6 \chi S_{d}\right)^{2}}{2 \lambda} \cdot\left\|\rho_{s}\right\|_{4}^{2-\frac{8}{3 d}}-\frac{\lambda}{\left|N_{d}\right|^{\frac{2(d+2)}{d}}}\right) \mathrm{d} s .
$$

Therefore, if

$$
\frac{\left(6 \chi S_{d}\right)^{2}}{2 \lambda} \cdot\left\|\rho_{0}\right\|_{4}^{2-\frac{8}{3 d}}-\frac{\lambda}{\left|N_{d}\right|^{\frac{2(d+2)}{d}}} \leq 0
$$

, i.e.,

$$
\left\|\rho_{0}\right\|_{4} \leq\left(\frac{2 \lambda^{2}}{\left|N_{d}\right|^{\frac{2(d+2)}{d}}\left(6 \chi S_{d}\right)^{2}}\right)^{\frac{3 d}{6 d-8}}
$$

then we conclude from (3.20) that $\left\|\rho_{t}\right\|_{4} \leq\left\|\rho_{0}\right\|_{4}$ for all $t \in\left[0, T_{0}\right]$ and that the unique solution may actually be extended to any finite time interval $[0, T]$.

Corollary 1 Let Assumption 1 hold with $m=2$ and the diffusion coefficients $v$ and $\sigma$ being spatial invariant [see (3.17)]. Assume $0 \leq \rho_{0} \in L^{1} \cap \mathcal{H}_{4}^{\frac{1}{2}}\left(\mathbb{R}^{d}\right)$ with $\left\|\rho_{0}\right\|_{1}=1$. There exists a constant $\kappa>0$ depending only on $\chi, \lambda$, and $d$ such that if $\left\|\rho_{0}\right\|_{4} \leq \kappa$, SPDE (1.3) admits a unique nonnegative solution in

$$
L_{\mathcal{F}^{W}}^{2}\left(0, T ; W^{1,2}\left(\mathbb{R}^{d}\right)\right) \cap L_{\mathcal{F}^{W}}^{4}\left(0, T ; W^{1,4}\left(\mathbb{R}^{d}\right)\right) \cap S_{\mathcal{F}^{W}}^{\infty}\left([0, T] ; L^{1} \cap L^{4}\left(\mathbb{R}^{d}\right)\right),
$$


for all $T>0$.

In Corollary 1, the constant $\kappa$ may be given as the right-hand side of (3.21) that is independent of $(T, \Lambda)$ and the global solution result with small initial value under $L^{4}$-norm seems to hold in a similar way as the deterministic counterparts (see Blanchet et al. 2006; Corrias et al. 2004; Biler 2010 for instance). The results in Theorem 3.2, Corollary 1 , and subsequent theorems, may be extended to general $L^{p}$-norms for $p>3$, which would not be discussed in this paper to avoid cumbersome arguments.

To explore the connections between the stochastic Keller-Segel Eq. (1.3) and associated SDEs of McKean-Vlasov type (1.7), we need stronger regularity of the solution.

Theorem 3.3 Let Assumption 1 hold with $m=3$. Suppose further $\rho_{0} \in L^{1} \cap W^{2,2}\left(\mathbb{R}^{d}\right)$. Then, for any $T>0$, there exists $\kappa>0$ depending only on $T, \Lambda, \lambda, \chi$ and $d$ such that if $\left\|\rho_{0}\right\|_{4} \leq \kappa, \operatorname{SPDE}(1.3)$ admits a unique nonnegative solution in

$$
\begin{aligned}
\mathbb{M}_{1}:= & L_{\mathcal{F} W}^{2}\left(0, T ; W^{3,2}\left(\mathbb{R}^{d}\right)\right) \cap S_{\mathcal{F}^{W}}^{2}\left([0, T] ; W^{2,2}\left(\mathbb{R}^{d}\right)\right) \\
& \cap L_{\mathcal{F}^{W}}^{4}\left(0, T ; W^{1,4}\left(\mathbb{R}^{d}\right)\right) \cap S_{\mathcal{F}^{W}}^{\infty}\left([0, T] ; L^{1} \cap L^{4}\left(\mathbb{R}^{d}\right)\right) .
\end{aligned}
$$

Proof Notice that $W^{2,2}\left(\mathbb{R}^{d}\right) \hookrightarrow \mathcal{H}_{4}^{\frac{1}{2}}\left(\mathbb{R}^{d}\right) \hookrightarrow L^{4}\left(\mathbb{R}^{d}\right)$ for $d=2$ or 3 . Comparing Theorems 3.3 and 3.2, we only need to prove that the obtained unique solution $\rho$ in Theorem 3.2 is also lying in $L_{\mathcal{F} W}^{2}\left(0, T ; W^{3,2}\left(\mathbb{R}^{d}\right)\right) \cap S_{\mathcal{F} W}^{2}\left([0, T] ; W^{2,2}\left(\mathbb{R}^{d}\right)\right)$. In fact, $\rho \in \mathbb{M}$ [defined in (3.4)] is the solution of the following linear SPDE:

$$
\left\{\begin{array}{l}
\mathrm{d} \rho_{t}=\left[\frac{1}{2} \sum_{i, j=1}^{d} D_{i j}\left(\rho_{t} \sum_{k=1}^{d^{\prime}}\left(v^{i k} \nu^{j k}+\sigma^{i k} \sigma^{j k}\right)\right)+\chi f_{t}\right] \mathrm{d} t \\
\quad-\sum_{i=1}^{d} D_{i}\left(\rho_{t} \sum_{k=1}^{d^{\prime}} \sigma^{i k}\right) \mathrm{d} W_{t}^{k} \\
\rho_{0} \text { is given, }
\end{array}\right.
$$

with

$$
f_{t}=-\nabla \cdot\left(\rho_{t} \nabla c_{t}\right)=-\nabla \rho_{t} \cdot \nabla c_{t}+\rho_{t}^{2}-\rho_{t} c_{t}
$$

As $\rho \in \mathbb{M}$, it follows that

$$
\begin{aligned}
\left\|f_{t}\right\|_{2} & =\left\|\nabla \cdot\left(\rho_{t} \nabla c_{t}\right)\right\|_{2} \leq\left\|\nabla c_{t}\right\|_{\infty}\left\|\nabla \rho_{t}\right\|_{2}+\left\|\rho_{t}\right\|_{4}^{2}+\left\|\rho_{t}\right\|_{2}\left\|c_{t}\right\|_{\infty} \\
& \leq S_{d}\|\rho\|_{4}\left\|\nabla \rho_{t}\right\|_{2}+\left\|\rho_{t}\right\|_{4}^{2}+S_{d}\left\|\rho_{t}\right\|_{2}\left\|\rho_{t}\right\|_{4} \leq S_{d}\|\rho\|_{4}\left\|\rho_{t}\right\|_{W^{1,2}}+\left\|\rho_{t}\right\|_{4}^{2},
\end{aligned}
$$

which indicates that

$$
\|f\|_{L_{\mathcal{F} W}^{2}\left(0, T ; L^{2}\right)}^{2} \leq 2 S_{d}^{2}\|\rho\|_{S_{\mathcal{F} W}^{\infty}\left([0, T] ; L^{4}\right)}^{2}\|\rho\|_{L_{\mathcal{F} W}^{2}\left(0, T ; W^{1,2}\right)}^{2}+2\|\rho\|_{L_{\mathcal{F} W}^{4}\left(0, T ; L^{4}\right)}^{4}<\infty .
$$


The $L^{p}$-theory of SPDE (see Krylov 1999, Theorem 5.1) and Theorem 3.2 imply that

$$
\rho \in L_{\mathcal{F}^{W}}^{2}\left(0, T ; W^{2,2}\left(\mathbb{R}^{d}\right)\right) \cap S_{\mathcal{F}^{W}}^{2}\left([0, T] ; W^{1,2}\left(\mathbb{R}^{d}\right)\right) \cap \mathbb{M} .
$$

Similarly, for $j=1, \ldots, d$, one has

$$
\left\|D_{j} f_{t}\right\|_{2} \leq C\left\|\rho_{t}\right\|_{W^{2,2}}\left\|\rho_{t}\right\|_{4}+C\left\|\rho_{t}\right\|_{W^{1,4}}\left\|\rho_{t}\right\|_{4},
$$

which together with (3.24) and (3.23) implies that

$$
\|f\|_{L_{\mathcal{F} W}^{2}\left(0, T ; W^{1,2}\right)}<\infty .
$$

Hence, applying the $L^{p}$-theory of SPDE (see Krylov 1999, Theorem 5.1) and Theorem 3.2 again, we conclude

$$
\begin{aligned}
& \rho \in L_{\mathcal{F}^{W}}^{2}\left(0, T ; W^{3,2}\left(\mathbb{R}^{d}\right)\right) \cap S_{\mathcal{F} W}^{2}\left([0, T] ; W^{2,2}\left(\mathbb{R}^{d}\right)\right) \\
& \quad \cap L_{\mathcal{F} W}^{4}\left(0, T ; W^{1,4}\left(\mathbb{R}^{d}\right)\right) \cap S_{\mathcal{F} W}^{\infty}\left([0, T] ; L^{1} \cap L^{4}\left(\mathbb{R}^{d}\right)\right)
\end{aligned}
$$

The proof is completed.

\section{Well-Posedness of the Nonlinear SDE}

Let us consider the following SDE:

$$
\left\{\begin{array}{l}
\mathrm{d} Y_{t}=\chi \nabla \mathcal{G} * \rho_{t}\left(Y_{t}\right) \mathrm{d} t+v_{t}\left(Y_{t}\right) \mathrm{d} B_{t}+\sigma_{t}\left(Y_{t}\right) \mathrm{d} W_{t}, \quad t>0 \\
\rho_{t} \text { is the conditional density of } Y_{t} \text { given } \mathcal{F}_{t}^{W} \\
Y_{0}=\zeta^{1}
\end{array}\right.
$$

where we take $B_{t}=B_{t}^{1}$ in this section as a $d^{\prime}$-dimensional Wiener process independent of $W_{t}$ and $\zeta^{1}$. In the following, we prove the well-posedness of the nonlinear SDE (4.1) which actually shares the same solvability as $\operatorname{SDE}(1.7)$ for each $i \in \mathbb{Z}^{+}$.

Theorem 4.1 (Well-posedness of the SDE) Under the same assumptions as in Theorem 3.3, let $\rho$ be the regular solution to the SPDE (1.3) obtained in Theorem 3.3. Then, the nonlinear SDE (4.1) has a unique strong solution $\left(Y_{t}\right)_{t \geq 0}$ with $\rho \in$ $S_{\mathcal{F} W}^{2}\left([0, T] ; W^{2,2}\left(\mathbb{R}^{d}\right)\right) \cap S_{\mathcal{F} W}^{\infty}\left([0, T] ; L^{4}\left(\mathbb{R}^{d}\right)\right)$ being its conditional density under filtration $\left(\mathcal{F}_{t}^{W}\right)_{t \in[0, T]}$.

Proof For the solution $\rho \in S_{\mathcal{F} W}^{2}\left(\left([0, T] ; W^{2,2}\left(\mathbb{R}^{d}\right)\right) \cap S_{\mathcal{F} W}^{\infty}\left([0, T] ; L^{4}\left(\mathbb{R}^{d}\right)\right)\right.$ of the $\operatorname{SPDE}$ (1.3) given in Theorem 3.3, by embedding theorems, we have

$$
\begin{gathered}
\nabla \mathcal{G} * \rho \in S_{\mathcal{F} W}^{2}\left([0, T] ; W^{3,2}\left(\mathbb{R}^{d}\right)\right) \cap S_{\mathcal{F}^{W}}^{\infty}\left([0, T] ; W^{1,4}\left(\mathbb{R}^{d}\right)\right) \\
\hookrightarrow S_{\mathcal{F} W}^{2}\left([0, T] ; W^{1, \infty}\left(\mathbb{R}^{d}\right)\right) \cap S_{\mathcal{F} W}^{\infty}\left([0, T] ; L^{\infty}\left(\mathbb{R}^{d}\right)\right)
\end{gathered}
$$


which ensures the existence and uniqueness of strong solution $\left(\bar{Y}_{t}\right)_{t \geq 0}$ to the following linear SDE:

$$
\left\{\begin{array}{l}
\mathrm{d} \bar{Y}_{t}=\chi \nabla \mathcal{G} * \rho_{t}\left(\bar{Y}_{t}\right) \mathrm{d} t+v_{t}\left(\bar{Y}_{t}\right) \mathrm{d} B_{t}+\sigma_{t}\left(\bar{Y}_{t}\right) \mathrm{d} W_{t}, \quad t>0, \\
\bar{Y}_{0}=\zeta^{1}
\end{array}\right.
$$

To prove that the conditional density given $\mathcal{F}_{t}^{W}$ of $\left(\bar{Y}_{t}\right)_{t \geq 0}$ exists and is the solution to SPDE (1.3), we need the following result on backward SPDE and associated probabilistic representation.

Lemma 4.1 Let Assumption 1 hold with $m=3, \rho \in S_{\mathcal{F} W}^{2}\left([0, T] ; W^{2,2}\left(\mathbb{R}^{d}\right)\right) \cap$ $S_{\mathcal{F} W}^{\infty}\left([0, T] ; L^{4}\left(\mathbb{R}^{d}\right)\right)$ and $T_{1} \in(0, T]$. Then, for each $G \in L^{2}\left(\Omega, \mathcal{F}_{T_{1}} ; W^{2,2}\left(\mathbb{R}^{d}\right)\right)$, the following backward SPDE:

$$
\left\{\begin{array}{l}
-\mathrm{d} u_{t}=\left[\frac{1}{2} \sum_{i, j=1}^{d} \sum_{k=1}^{d^{\prime}}\left(v_{t}^{i k} v_{t}^{j, k}+\sigma_{t}^{i k} \sigma_{t}^{j k}\right) D_{i j} u_{t}+\chi \sum_{i=1}^{d} D_{i} \mathcal{G} * \rho_{t} D_{i} u_{t}+\sum_{i=1}^{d} \sum_{k=1}^{d^{\prime}} \sigma_{t}^{i k} D_{i} \psi_{t}^{k}\right] \mathrm{d} t \\
-\sum_{k=1}^{d^{\prime}} \psi_{t}^{k} \mathrm{~d} W_{t}^{k}, \\
u_{T_{1}}=G
\end{array}\right.
$$

admits a unique solution

$$
(u, \psi) \in\left(L_{\mathcal{F}^{W}}^{2}\left(0, T ; W^{3,2}\left(\mathbb{R}^{d}\right)\right) \cap S_{\mathcal{F}^{W}}^{2}\left([0, T] ; W^{2,2}\left(\mathbb{R}^{d}\right)\right)\right) \times L_{\mathcal{F}^{W}}^{2}\left(0, T ; W^{2,2}\left(\mathbb{R}^{d}\right)\right),
$$

i.e., for any $\varphi \in C_{c}^{2}\left(\mathbb{R}^{d}\right)$, there holds for each $t \in\left[0, T_{1}\right]$,

$$
\begin{aligned}
\left\langle u_{t}, \varphi\right\rangle= & \langle\varphi, G\rangle \\
& +\int_{t}^{T_{1}}\left\langle\varphi, \frac{1}{2} \sum_{i, j=1}^{d} \sum_{k=1}^{d^{\prime}}\left(v_{s}^{i k} v_{s}^{j k}+\sigma_{s}^{i k} \sigma_{s}^{j k}\right) D_{i j} u_{s}+\chi \sum_{i=1}^{d} D_{i} \mathcal{G} * \rho_{s} D_{i} u_{s}+\sum_{i=1}^{d} \sum_{k=1}^{d^{\prime}} \sigma_{s}^{i k} D_{i} \psi_{s}^{k}\right\rangle \mathrm{d} s \\
& -\int_{t}^{T_{1}} \sum_{k=1}^{d^{\prime}}\left\langle\varphi, \psi_{s}^{k}\right\rangle \mathrm{d} W_{s}^{k}, \quad \text { a.s. }
\end{aligned}
$$

Moreover, for this solution, we have

$$
u_{t}(y)=\mathbb{E}\left[G\left(\bar{Y}_{T_{1}}\right) \mid \bar{Y}_{t}=y, \mathcal{F}_{t}^{W}\right], \quad \text { a.s.foranyt } \in\left[0, \mathrm{~T}_{1}\right] .
$$

For each $T_{1} \in(0, T]$, take an arbitrary $\xi \in L^{\infty}\left(\Omega, \mathcal{F}_{T_{1}}\right)$ and $\phi \in C_{c}^{\infty}\left(\mathbb{R}^{d}\right)$. In view of the SPDE (1.3), applying the Itô formula to $\left\langle u_{t}, \rho_{t}\right\rangle$ [the duality analysis on the (1.3) and (4.4) as in Du et al. (2011), Zhou (1992)] gives

$$
\left\langle u_{0}, \rho_{0}\right\rangle=\left\langle\xi \phi, \rho_{T_{1}}\right\rangle-\int_{0}^{T_{1}} \sum_{i=1}^{d} \sum_{k=1}^{d^{\prime}}\left\langle u_{t}, D_{i}\left(\sigma_{t}^{i k} \rho_{t}\right)\right\rangle \mathrm{d} W_{t}^{k}-\int_{0}^{T_{1}} \sum_{k=1}^{d^{\prime}}\left\langle\rho_{t}, \psi_{t}^{k}\right\rangle \mathrm{d} W_{t}^{k}, \quad \text { a.s. }
$$


where $(u, \psi)$ is the solution in Lemma 4.1 with $G=\xi \phi$. Then, we have by taking expectations on both sides,

$$
\left\langle u_{0}, \rho_{0}\right\rangle=\mathbb{E}\left[\left\langle\xi \phi, \rho_{T_{1}}\right\rangle\right]=\mathbb{E}\left[\xi\left\langle\phi, \rho_{T_{1}}\right\rangle\right]
$$

On the other hand, in view of the probabilistic representation (4.5), we have

$$
\left\langle u_{0}, \rho_{0}\right\rangle=\int_{\mathbb{R}^{d}} \mathbb{E}\left[G\left(\bar{Y}_{T_{1}}\right) \mid \bar{Y}_{0}=y, \mathcal{F}_{0}^{W}\right] \rho_{0}(y) \mathrm{d} y=\mathbb{E}\left[\xi \phi\left(\bar{Y}_{T_{1}}\right)\right]=\mathbb{E}\left[\xi \mathbb{E}\left[\phi\left(\bar{Y}_{T_{1}}\right) \mid \mathcal{F}_{T_{1}}^{W}\right]\right] .
$$

Therefore,

$$
\mathbb{E}\left[\xi\left\langle\phi, \rho_{T_{1}}\right\rangle\right]=\mathbb{E}\left[\xi \mathbb{E}\left[\phi\left(\bar{Y}_{T_{1}}\right) \mid \mathcal{F}_{T_{1}}^{W}\right]\right]
$$

which by the arbitrariness of $\left(T_{1}, \xi, \phi\right)$ implies that $\rho_{t}$ is the conditional density of $\bar{Y}_{t}$ given $\mathcal{F}_{t}^{W}$ for each $t \in[0, T]$ and shows the existence of strong solution to SDE (4.1). In fact, this also means that each strong solution of SDE (4.1) with $\rho \in S_{\mathcal{F} W}^{2}\left([0, T] ; W^{2,2}\left(\mathbb{R}^{d}\right)\right) \cap S_{\mathcal{F} W}^{\infty}\left([0, T] ; L^{4}\left(\mathbb{R}^{d}\right)\right)$ must have the conditional density $\rho$ being the solution to $\operatorname{SPDE}(1.3)$, and thus, the strong solution is unique. We complete the proof.

Proof of Lemma 4.1 Embedding theorem gives (4.2) which by the $L^{2}$-theory of backward SPDE (see Du et al. 2011; Zhou 1992) implies that backward SPDE (4.4) has a unique solution $(u, \psi) \in\left(L_{\mathcal{F} W}^{2}\left(0, T ; W^{1,2}\left(\mathbb{R}^{d}\right)\right) \cap S_{\mathcal{F} W}^{2}\left([0, T] ; L^{2}\left(\mathbb{R}^{d}\right)\right)\right) \times$ $L_{\mathcal{F} W}^{2}\left(0, T ; L^{2}\left(\mathbb{R}^{d}\right)\right) .{ }^{4}$ Then, we need to show that the solution $(u, \psi)$ has higher regularity as it is done in the proof of Theorem 3.3. In fact, we have for each $i=1, \ldots, d$,

$$
\left\|D_{i} \mathcal{G} * \rho_{s} D_{i} u_{s}\right\|_{2} \leq\left\|D_{i} \mathcal{G} * \rho_{s}\right\|_{\infty}\left\|D_{i} u_{s}\right\|_{2} \leq S_{d}\left\|\rho_{s}\right\|_{4}\left\|D_{i} u_{s}\right\|_{2},
$$

and thus, $D_{i} \mathcal{G} * \rho D_{i} u \in L_{\mathcal{F} W}^{2}\left(0, T_{1} ; L^{2}\right)$, which by $L^{2}$-theory of backward SPDE indicated further

$$
(u, \psi) \in\left(L_{\mathcal{F}^{W}}^{2}\left(0, T ; W^{2,2}\left(\mathbb{R}^{d}\right)\right) \cap S_{\mathcal{F}^{W}}^{2}\left([0, T] ; W^{1,2}\left(\mathbb{R}^{d}\right)\right)\right) \times L_{\mathcal{F}^{W}}^{2}\left(0, T ; W^{1,2}\left(\mathbb{R}^{d}\right)\right) .
$$

Taking derivatives gives further

$$
\begin{aligned}
\left\|D_{j}\left(D_{i} \mathcal{G} * \rho_{s} D_{i} u_{s}\right)\right\|_{2} & \leq\left\|D_{i j} \mathcal{G} * \rho_{s} D_{i} u_{s}\right\|_{2}+\left\|D_{i} \mathcal{G} * \rho_{s} D_{i j} u_{s}\right\|_{2} \\
& \leq\left\|D_{i j} \mathcal{G} * \rho_{s}\right\|_{4}\left\|D_{i} u_{s}\right\|_{4}+\left\|D_{i} \mathcal{G} * \rho_{s}\right\|_{\infty}\left\|D_{i j} u_{s}\right\|_{2} \\
& \leq S_{d}\left\|\rho_{s}\right\|_{4}\left\|D_{i} u_{s}\right\|_{2}^{1 / 4}\left\|D_{i} u_{s}\right\|_{6}^{3 / 4}+S_{d}\left\|\rho_{s}\right\|_{4}\left\|D_{i j} u_{s}\right\|_{2} \\
& \leq\left\|\rho_{s}\right\|_{4}\left\|u_{s}\right\|_{W^{2,2}}+S_{d}\left\|\rho_{s}\right\|_{4}\left\|u_{s}\right\|_{W^{2,2}},
\end{aligned}
$$

\footnotetext{
4 The fact $u \in S_{\mathcal{F} W}^{2}\left([0, T] ; L^{2}\left(\mathbb{R}^{d}\right)\right)$ is not claimed in Du et al. (2011), Zhou (1992), but it follows straightforwardly from Ren et al. (2007, Theorem A.2) for Itô's formula of square norms. It is similar in the relation (4.6).
} 
and thus, $D_{i} \mathcal{G} * \rho_{s} D_{i} u_{s} \in L_{\mathcal{F} W}^{2}\left(0, T_{1} ; W^{1,2}\left(\mathbb{R}^{d}\right)\right), i=1, \ldots, d$. Applying the $L^{2}$ theory again, we arrive at

$$
(u, \psi) \in\left(L_{\mathcal{F} W}^{2}\left(0, T ; W^{3,2}\left(\mathbb{R}^{d}\right)\right) \cap S_{\mathcal{F} W}^{2}\left([0, T] ; W^{2,2}\left(\mathbb{R}^{d}\right)\right)\right) \times L_{\mathcal{F} W}^{2}\left(0, T ; W^{2,2}\left(\mathbb{R}^{d}\right)\right) .
$$

W.1.o.g., we prove the probabilistic representation (4.5) for the case when $t=0$. In fact, a straightforward application of Yang and Tang (2013, Theorem 3.1) yields that

$$
u_{0}(y)=G\left(\bar{Y}_{T_{1}}\right)-\int_{0}^{T_{1}}\left(\sum_{k}^{d^{\prime}} \psi_{s}^{k}\left(\bar{Y}_{s}\right)+\sum_{i=1}^{d} \sigma_{s}^{i k}\left(\bar{Y}_{s}\right) D_{i} u_{s}\left(\bar{Y}_{s}\right)\right) \mathrm{d} W_{s}^{k}, \quad \text { a.s. }
$$

Noticing that by embedding theorem it holds that $L_{\mathcal{F} W}^{2}\left(0, T ; W^{2,2}\left(\mathbb{R}^{d}\right)\right) \hookrightarrow$ $L_{\mathcal{F} W}^{2}\left(0, T ; C^{1 / 4}\left(\mathbb{R}^{d}\right)\right)$, we may easily check that the stochastic integral in the above equality is mean-zero. Therefore, we have $u_{0}(y)=\mathbb{E}\left[G\left(\bar{Y}_{T_{1}}\right) \mid \bar{Y}_{0}=y, \mathcal{F}_{0}^{W}\right]$ by taking conditional expectation on both sides. For general $t \in\left(0, T_{1}\right]$, the proof of (4.5) follows similarly.

\section{Mean-Field Limit of the Particle System (1.5) Toward the Stochastic KS Equation (1.3)}

To prove the mean-field limit, we recall the following auxiliary stochastic dynamics $\left\{\left(Y_{t}^{i}\right)_{t \geq 0}\right\}_{i=1}^{N}$ as defined in (1.7)

$$
\left\{\begin{array}{l}
\mathrm{d} Y_{t}^{i}=\chi \nabla \mathcal{G} * \rho_{t}\left(Y_{t}^{i}\right) \mathrm{d} t+v_{t}\left(Y_{t}^{i}\right) \mathrm{d} B_{t}^{i}+\sigma_{t}\left(Y_{t}^{i}\right) \mathrm{d} W_{t}, \quad t>0, \quad i=1, \ldots, N \\
\rho_{t} \text { is the conditional density of } Y_{t}^{i} \text { given } \mathcal{F}_{t}^{W} \text { for all } i=1, \ldots, N \\
Y_{0}^{i}=\zeta^{i}
\end{array}\right.
$$

This means that $\left\{\left(Y_{t}^{i}\right)_{t \geq 0}\right\}_{i=1}^{N}$ are $N$ copies of solutions to the nonlinear SDE (4.1), and they are conditional i.i.d. given $W_{t}$. We will also use the regularized version

$$
\left\{\begin{array}{l}
\mathrm{d} Y_{t}^{i, \varepsilon}=\chi \nabla \mathcal{G}_{\varepsilon} * \rho_{t}^{\varepsilon}\left(Y_{t}^{i, \varepsilon}\right) \mathrm{d} t+v_{t}\left(Y_{t}^{i, \varepsilon}\right) \mathrm{d} B_{t}^{i}+\sigma_{t}\left(Y_{t}^{i, \varepsilon}\right) \mathrm{d} W_{t}, \quad t>0, \quad i=1, \ldots, N \\
\rho_{t}^{\varepsilon} \text { is the conditional density of } Y_{t}^{i, \varepsilon} \text { given } \mathcal{F}_{t}^{W} \text { for all } i=1, \ldots, N \\
Y_{0}^{i, \varepsilon}=Y_{0}^{i}=\zeta^{i}
\end{array}\right.
$$

with $\rho_{t}^{\varepsilon}$ satisfying the following regularized stochastic KS equation

$$
\begin{cases}\mathrm{d} \rho_{t}^{\varepsilon}= & \frac{1}{2} \sum_{i, j=1}^{d} D_{i j}\left(\rho_{t}^{\varepsilon} \sum_{k=1}^{d^{\prime}}\left(v_{t}^{i k} v_{t}^{j k}+\sigma_{t}^{i k} \sigma_{t}^{j k}\right)\right) \mathrm{d} t-\chi \nabla \cdot\left(\nabla\left(\mathcal{G}_{\varepsilon} * \rho_{t}^{\varepsilon}\right) \rho_{t}^{\varepsilon}\right) \mathrm{d} t \\ & -\sum_{i=1}^{d} D_{i}\left(\rho_{t}^{\varepsilon} \sum_{k=1}^{d^{\prime}} \sigma_{t}^{i k} \mathrm{~d} W_{t}^{k}\right), \\ \rho_{0}^{\varepsilon}=\rho_{0}, & \end{cases}
$$


Indeed, following the same arguments as in Sects. 3-4, we obtain the well-posedness of the regularized system (5.2) and Eq. (5.3). Next, we estimate the difference of the solutions. Set $e_{t}^{\varepsilon}=\rho_{t}^{\varepsilon}-\rho_{t}$ for $t \in[0, T]$ with $e_{0}^{\varepsilon}=0$. Following the same computation as in (3.14), one has

$$
\begin{aligned}
\left\|e_{t}^{\varepsilon}\right\|_{4}^{4} \leq & -6 \lambda \int_{0}^{t}\left\|e_{s}^{\varepsilon} \nabla e_{s}^{\varepsilon}\right\|_{2}^{2} \mathrm{~d} s+2 \lambda \int_{0}^{t}\left\|e_{s}^{\varepsilon} \nabla e_{s}^{\varepsilon}\right\|_{2}^{2} \mathrm{~d} s+\frac{\left(12 \Lambda^{2}\right)^{2}}{2 \lambda} \int_{0}^{t}\left\|e_{s}^{\varepsilon}\right\|_{4}^{4} \mathrm{~d} s \\
& +\int_{0}^{t} 12 \chi\left\langle\left|e_{s}^{\varepsilon}\right|^{2} \nabla e_{s}^{\varepsilon},\left(\nabla \mathcal{G}_{\varepsilon} * \rho_{s}^{\varepsilon}\right) \rho_{s}^{\varepsilon}-\left(\nabla \mathcal{G} * \rho_{s}\right) \rho_{s}\right\rangle \mathrm{d} s, \quad \text { a.s.. }
\end{aligned}
$$

Notice that

$$
\begin{aligned}
& 12 \chi\left\langle\left|e_{s}^{\varepsilon}\right|^{2} \nabla e_{s}^{\varepsilon},\left(\nabla \mathcal{G}_{\varepsilon} * \rho_{s}^{\varepsilon}\right) \rho_{s}^{\varepsilon}-\left(\nabla \mathcal{G} * \rho_{s}\right) \rho_{s}\right\rangle \\
& \quad=12 \chi\left\langle\left|e_{s}^{\varepsilon}\right|^{2} \nabla e_{s}^{\varepsilon}, \nabla \mathcal{G} * e_{s}^{\varepsilon} \rho_{s}^{\varepsilon}+\nabla \mathcal{G} * \rho_{s} e_{s}^{\varepsilon}\right\rangle+12 \chi\left\langle\left|e_{s}^{\varepsilon}\right|^{2} \nabla e_{s}^{\varepsilon},\left(\nabla \mathcal{G}_{\varepsilon}-\nabla \mathcal{G}\right) * \rho_{s}^{\varepsilon} \rho_{s}^{\varepsilon}\right\rangle .
\end{aligned}
$$

Similar to the computation in (3.15), one obtains

$$
12 \chi\left\langle\left|e_{s}^{\varepsilon}\right|^{2} \nabla e_{s}^{\varepsilon}, \nabla \mathcal{G} * e_{s}^{\varepsilon} \rho_{s}^{\varepsilon}+\nabla \mathcal{G} * \rho_{s} e_{s}^{\varepsilon}\right\rangle \leq 2 \lambda\left\|e_{s}^{\varepsilon} \nabla e_{s}^{\varepsilon}\right\|_{2}^{2}+C(T, \chi, \lambda, \Lambda, d)\left\|e_{s}^{\varepsilon}\right\|_{4}^{4} .
$$

On the other hand, we compute

$$
12 \chi\left\langle\left|e_{s}^{\varepsilon}\right|^{2} \nabla e_{s}^{\varepsilon},\left(\nabla \mathcal{G}_{\varepsilon}-\nabla \mathcal{G}\right) * \rho_{s}^{\varepsilon} \rho_{s}^{\varepsilon}\right\rangle \leq 12 \chi\left\|e_{s}^{\varepsilon} \nabla e_{s}^{\varepsilon}\right\|_{2}\left\|\left(\nabla \mathcal{G}_{\varepsilon}-\nabla \mathcal{G}\right) * \rho_{s}^{\varepsilon}\right\|_{\infty}\left\|e_{s}^{\varepsilon}\right\|_{4}\left\|\rho_{s}^{\varepsilon}\right\|_{4} .
$$

Notice that

$$
\begin{aligned}
& \left|\left(\nabla \mathcal{G}_{\varepsilon}-\nabla \mathcal{G}\right) * \rho_{s}^{\varepsilon}\right|(x) \\
& \quad=\left|\psi_{\varepsilon} *\left(\nabla \mathcal{G} * \rho_{s}^{\varepsilon}\right)-\nabla \mathcal{G} * \rho_{s}^{\varepsilon}\right|(x)=\left|\int_{\mathbb{R}^{d}} \psi_{\varepsilon}(y)\left[\nabla \mathcal{G} * \rho_{s}^{\varepsilon}(x-y)-\nabla \mathcal{G} * \rho_{s}^{\varepsilon}(x)\right] \mathrm{d} y\right| \\
& \quad \leq\left\|\nabla \mathcal{G} * \rho_{s}^{\varepsilon}\right\|_{W^{1, \infty}} \int_{\mathbb{R}^{d}}|y| \psi_{\varepsilon}(y) \mathrm{d} y \leq C \varepsilon\left\|\nabla \mathcal{G} * \rho_{s}^{\varepsilon}\right\|_{W^{1, \infty}} \leq C \varepsilon\left\|\rho_{s}^{\varepsilon}\right\|_{W^{1,4}},
\end{aligned}
$$

where $C$ depends only on $T, \chi, \lambda, \Lambda$, and $d$. Then, one has

$$
12 \chi\left\langle\left|e_{s}^{\varepsilon}\right|^{2} \nabla e_{s}^{\varepsilon},\left(\nabla \mathcal{G}_{\varepsilon}-\nabla \mathcal{G}\right) * \rho_{s}^{\varepsilon} \rho_{s}^{\varepsilon}\right\rangle \leq 2 \lambda\left\|e_{s}^{\varepsilon} \nabla e_{s}^{\varepsilon}\right\|_{2}^{2}+C \varepsilon^{2}\left\|\rho_{s}^{\varepsilon}\right\|_{W^{1,4}}^{2}\left\|e_{s}^{\varepsilon}\right\|_{4}^{2}\left\|\rho_{s}^{\varepsilon}\right\|_{4}^{2},
$$

and thus

$$
\begin{aligned}
& 12 \chi\left\langle\left|e_{s}^{\varepsilon}\right|^{2} \nabla e_{s}^{\varepsilon},\left(\nabla \mathcal{G}_{\varepsilon} * \rho_{s}^{\varepsilon}\right) \rho_{s}^{\varepsilon}-\left(\nabla \mathcal{G} * \rho_{s}\right) \rho_{s}\right\rangle \\
& \leq 4 \lambda\left\|e_{s}^{\varepsilon} \nabla e_{s}^{\varepsilon}\right\|_{2}^{2}+C \varepsilon^{2}\left\|\rho_{s}^{\varepsilon}\right\|_{W^{1,4}}^{2}\left\|e_{s}^{\varepsilon}\right\|_{4}^{2}\left\|\rho_{s}^{\varepsilon}\right\|_{4}^{2}+C\left\|e_{s}^{\varepsilon}\right\|_{4}^{4} .
\end{aligned}
$$


It follows from (5.4) that

$$
\left\|e_{t}^{\varepsilon}\right\|_{4}^{4} \leq C_{1} \int_{0}^{t}\left\|e_{s}^{\varepsilon}\right\|_{4}^{4} \mathrm{~d} s+C_{2} \varepsilon^{2} \int_{0}^{t}\left\|\rho_{s}^{\varepsilon}\right\|_{W^{1,4}}^{2}\left\|e_{s}^{\varepsilon}\right\|_{4}^{2}\left\|\rho_{s}^{\varepsilon}\right\|_{4}^{2} \mathrm{~d} s,
$$

where $C_{1}, C_{2}$ depend only on $T, \chi, \lambda, \Lambda$, and $d$. By Gronwall's inequality, we have

$$
\sup _{t \in[0, T]}\left\|e_{t}^{\varepsilon}\right\|_{4}^{4} \leq\left[C_{2} \varepsilon^{2} \sup _{t \in[0, T]}\left\{\left\|e_{t}^{\varepsilon}\right\|_{4}^{2}\left\|\rho_{t}^{\varepsilon}\right\|_{4}^{2}\right\} \int_{0}^{T}\left\|\rho_{s}^{\varepsilon}\right\|_{W^{1,4}}^{2} \mathrm{~d} s\right] \exp \left(C_{1} T\right) \quad \text { a.s. }
$$

This leads to

$$
\begin{aligned}
& \left\|\rho^{\varepsilon}-\rho\right\|_{S_{\mathcal{F} W}^{2}\left([0, T] ; L^{4}\left(\mathbb{R}^{d}\right)\right)} \\
& \quad \leq C\left(C_{1}, C_{2}, T,\|\rho\|_{S_{\mathcal{F} W}^{\infty}\left([0, T] ; L^{4}\left(\mathbb{R}^{d}\right)\right)},\left\|\rho^{\varepsilon}\right\|_{S_{\mathcal{F} W}^{\infty}\left([0, T] ; L^{4}\left(\mathbb{R}^{d}\right)\right)},\left\|\rho^{\varepsilon}\right\|_{L_{\mathcal{F} W}^{2}\left([0, T] ; W^{1,4}\left(\mathbb{R}^{d}\right)\right)}\right) \varepsilon \\
& \quad \leq C(T, \chi, \lambda, \Lambda, d) \cdot \varepsilon
\end{aligned}
$$

where we have used the fact that the quantities $\|\rho\|_{S_{\mathcal{F} W}^{\infty}\left([0, T] ; L^{4}\left(\mathbb{R}^{d}\right)\right)}$, $\left\|\rho^{\varepsilon}\right\|_{S_{\mathcal{F} W}^{\infty}\left([0, T] ; L^{4}\left(\mathbb{R}^{d}\right)\right)}$, and $\left\|\rho^{\varepsilon}\right\|_{L_{\mathcal{F} W}^{2}\left([0, T] ; W^{1,4}\left(\mathbb{R}^{d}\right)\right)}$ depend only on $T, \chi, \lambda, \Lambda$, and $d$, independent of $\varepsilon$.

Our main theorem of mean-field limit states that the mean-field dynamics $\left\{\left(Y_{t}^{i, \varepsilon}\right)_{t \geq 0}\right\}_{i=1}^{N}$ well approximate the regularized interacting particle system $\left\{\left(X_{t}^{i, \varepsilon}\right)_{t \geq 0}\right\}_{i=1}^{N}$ in (1.5).

Theorem 5.1 Under the same assumptions as in Theorem 3.3, let $\left\{\left(X_{t}^{i, \varepsilon}\right)_{t \geq 0}\right\}_{i=1}^{N}$ and $\left\{\left(Y_{t}^{i, \varepsilon}\right)_{t \geq 0}\right\}_{i=1}^{N}$ satisfy the interacting particle system (1.5) and the mean-field dynamics (5.2), respectively. Then, for any fixed $0<\delta \ll 1$, such that $\varepsilon^{-d} \leq \delta \ln (N)$ and $C \delta<1$ it holds that

$$
\sup _{t \in[0, T]} \sup _{i=1, \ldots, N} \mathbb{E}\left[\left|X_{t}^{i, \varepsilon}-Y_{t}^{i, \varepsilon}\right|^{2}\right] \leq C \frac{(\delta \ln (N))^{\frac{2 d-2}{d}}}{N^{1-C \delta}},
$$

where $C$ is a constant depending only on $\chi, T, d, d^{\prime}$ and $\Lambda$.

Proof Applying Itô's formula yields that

$$
\begin{aligned}
\mid X_{t}^{i, \varepsilon} & -\left.Y_{t}^{i, \varepsilon}\right|^{2} \\
= & \int_{0}^{t} 2 \chi\left(X_{s}^{i, \varepsilon}-Y_{s}^{i, \varepsilon}\right) \cdot\left(\frac{1}{N-1} \sum_{j \neq i}^{N} \nabla \mathcal{G}_{\varepsilon}\left(X_{s}^{i, \varepsilon}-X_{s}^{j, \varepsilon}\right)-\nabla \mathcal{G}_{\varepsilon} * \rho_{s}^{\varepsilon}\left(Y_{s}^{i, \varepsilon}\right)\right) \mathrm{d} s \\
& +\int_{0}^{t} 2\left(X_{s}^{i, \varepsilon}-Y_{s}^{i, \varepsilon}\right) \cdot\left(v_{s}\left(X_{s}^{i, \varepsilon}\right)-v_{s}\left(Y_{s}^{i, \varepsilon}\right)\right) \mathrm{d} B_{s}^{i}
\end{aligned}
$$




$$
\begin{aligned}
& +\int_{0}^{t} 2\left(X_{s}^{i, \varepsilon}-Y_{s}^{i, \varepsilon}\right) \cdot\left(\sigma_{s}\left(X_{s}^{i, \varepsilon}\right)-\sigma_{s}\left(Y_{s}^{i, \varepsilon}\right)\right) \mathrm{d} W_{s} \\
& +\int_{0}^{t} \sum_{j}^{d} \sum_{k=1}^{d^{\prime}}\left(v_{s}^{j k}\left(X_{s}^{i, \varepsilon}\right)-v_{s}^{j k}\left(Y_{s}^{i, \varepsilon}\right)\right)^{2} \mathrm{~d} s \\
& +\int_{0}^{t} \sum_{j}^{d} \sum_{k=1}^{d^{\prime}}\left(\sigma_{s}^{j k}\left(X_{s}^{i, \varepsilon}\right)-\sigma_{s}^{j k}\left(Y_{s}^{i, \varepsilon}\right)\right)^{2} \mathrm{~d} s .
\end{aligned}
$$

Taking expectations on both sides, one has

$$
\begin{aligned}
\mathbb{E}\left[\left|X_{t}^{i, \varepsilon}-Y_{t}^{i, \varepsilon}\right|^{2}\right] \\
\leq \mathbb{E}\left[\int_{0}^{t} 2 \chi\left(X_{s}^{i, \varepsilon}-Y_{s}^{i, \varepsilon}\right) \cdot\left(\frac{1}{N-1} \sum_{j \neq i}^{N} \nabla \mathcal{G}_{\varepsilon}\left(X_{s}^{i, \varepsilon}-X_{s}^{j, \varepsilon}\right)-\nabla \mathcal{G}_{\varepsilon} * \rho_{s}^{\varepsilon}\left(Y_{s}^{i, \varepsilon}\right)\right) \mathrm{d} s\right] \\
\quad+C\left(d, d^{\prime}, \Lambda\right) \int_{0}^{t} \mathbb{E}\left[\left|X_{s}^{i, \varepsilon}-Y_{s}^{i, \varepsilon}\right|^{2}\right] \mathrm{d} s
\end{aligned}
$$

where we have used the fact that

$$
\begin{aligned}
\mathbb{E} & {\left[\int_{0}^{t} 2\left(X_{s}^{i, \varepsilon}-Y_{s}^{i, \varepsilon}\right) \cdot\left(v_{s}\left(X_{s}^{i, \varepsilon}\right)-v_{s}\left(Y_{s}^{i, \varepsilon}\right)\right) \mathrm{d} B_{s}^{i}\right] } \\
& =\mathbb{E}\left[\int_{0}^{t} 2\left(X_{s}^{i, \varepsilon}-Y_{s}^{i, \varepsilon}\right) \cdot\left(\sigma_{s}\left(X_{s}^{i, \varepsilon}\right)-\sigma_{s}\left(Y_{s}^{i, \varepsilon}\right)\right) \mathrm{d} W_{s}\right]=0,
\end{aligned}
$$

and (ii) in Assumption 1.

To continue, we split the error

$\mathbb{E}\left[\int_{0}^{t} 2 \chi\left(X_{s}^{i, \varepsilon}-Y_{s}^{i, \varepsilon}\right) \cdot\left(\frac{1}{N-1} \sum_{j \neq i}^{N} \nabla \mathcal{G}_{\varepsilon}\left(X_{s}^{i, \varepsilon}-X_{s}^{j, \varepsilon}\right)-\nabla \mathcal{G}_{\varepsilon} * \rho_{s}^{\varepsilon}\left(Y_{s}^{i, \varepsilon}\right)\right) \mathrm{d} s\right]$

into three parts. Notice that

$$
\begin{aligned}
& \frac{1}{N-1} \sum_{j \neq i}^{N} \nabla \mathcal{G}_{\varepsilon}\left(X_{s}^{i, \varepsilon}-X_{s}^{j, \varepsilon}\right)-\nabla \mathcal{G}_{\varepsilon} * \rho_{s}^{\varepsilon}\left(Y_{s}^{i, \varepsilon}\right) \\
& =\frac{1}{N-1}\left(\sum_{j \neq i}^{N} \nabla \mathcal{G}_{\varepsilon}\left(X_{s}^{i, \varepsilon}-X_{s}^{j, \varepsilon}\right)-\sum_{j \neq i}^{N} \nabla \mathcal{G}_{\varepsilon}\left(Y_{s}^{i, \varepsilon}-Y_{s}^{j, \varepsilon}\right)\right) \\
& \quad+\frac{1}{N-1} \sum_{j \neq i}^{N} \nabla \mathcal{G}_{\varepsilon}\left(Y_{s}^{i, \varepsilon}-Y_{s}^{j, \varepsilon}\right)-\nabla \mathcal{G}_{\varepsilon} * \rho_{s}^{\varepsilon}\left(Y_{s}^{i, \varepsilon}\right) \\
& =: I_{11}^{s}+I_{12}^{s} .
\end{aligned}
$$


First, we compute

$$
\begin{aligned}
\int_{0}^{t} 2 \chi\left(X_{s}^{i, \varepsilon}-Y_{s}^{i, \varepsilon}\right) \cdot I_{11}^{s} \mathrm{~d} s & \leq 2 \chi \int_{0}^{t}\left|X_{s}^{i, \varepsilon}-Y_{s}^{i, \varepsilon}\right| \frac{1}{N-1}\left\|\nabla \mathcal{G}_{\varepsilon}\right\|_{W^{1, \infty}} \sum_{j=1}^{N}\left|X_{s}^{j, \varepsilon}-Y_{s}^{j, \varepsilon}\right| \mathrm{d} s \\
& \leq \frac{C \varepsilon^{-d}}{N-1} \int_{0}^{t} \sum_{j=1}^{N}\left|X_{s}^{j, \varepsilon}-Y_{s}^{j, \varepsilon}\right|^{2} \mathrm{~d} s
\end{aligned}
$$

which leads to

$$
\begin{aligned}
\mathbb{E}\left[\int_{0}^{t} 2 \chi\left(X_{s}^{i, \varepsilon}-Y_{s}^{i, \varepsilon}\right) \cdot I_{11}^{s} \mathrm{~d} s\right] & \leq \frac{C \varepsilon^{-d}}{N-1} \int_{0}^{t} \sum_{j=1}^{N} \mathbb{E}\left[\left|X_{s}^{j, \varepsilon}-Y_{s}^{j, \varepsilon}\right|^{2}\right] \mathrm{d} s \\
& \leq C \varepsilon^{-d} \int_{0}^{t} \sup _{i=1, \ldots, N} \mathbb{E}\left[\left|X_{s}^{i, \varepsilon}-Y_{s}^{i, \varepsilon}\right|^{2}\right] \mathrm{d} s
\end{aligned}
$$

where $C$ depends only on $\chi$ and $d$.

To estimate the second term, we rewrite

$$
I_{12}^{S}=\frac{1}{N-1} \sum_{j \neq i}^{N}\left(\nabla \mathcal{G}_{\varepsilon}\left(Y_{s}^{i, \varepsilon}-Y_{s}^{j, \varepsilon}\right)-\nabla \mathcal{G}_{\varepsilon} * \rho_{s}^{\varepsilon}\left(Y_{s}^{i, \varepsilon}\right)\right)=: \frac{1}{N-1} \sum_{j \neq i}^{N} Z_{j}^{i},
$$

where

$$
Z_{j}^{i}=\nabla \mathcal{G}_{\varepsilon}\left(Y_{s}^{i, \varepsilon}-Y_{s}^{j, \varepsilon}\right)-\nabla \mathcal{G}_{\varepsilon} * \rho_{s}^{\varepsilon}\left(Y_{s}^{i, \varepsilon}\right), \quad j \neq i .
$$

It is easy to check that

$$
\mathbb{E}\left[Z_{j}^{i} \mid \mathcal{F}_{t}^{W}, Y_{s}^{i, \varepsilon}\right]=\nabla \mathcal{G}_{\varepsilon} * \rho_{s}^{\varepsilon}\left(Y_{s}^{i, \varepsilon}\right)-\nabla \mathcal{G}_{\varepsilon} * \rho_{s}^{\varepsilon}\left(Y_{s}^{i, \varepsilon}\right)=0
$$

since $\left\{Y_{s}^{j, \varepsilon}\right\}_{j=1}^{N}$ are conditional i.i.d. with common conditional density $\rho_{s}^{\varepsilon}$ given $\mathcal{F}_{t}^{W}$. Thus, one concludes that

$$
\begin{aligned}
\mathbb{E}\left[\left|I_{12}^{s}\right|^{2}\right] & =\frac{1}{(N-1)^{2}} \mathbb{E}\left[\left(\sum_{j \neq i}^{N} Z_{j}^{i}\right)\left(\sum_{k \neq i}^{N} Z_{k}^{i}\right)\right] \\
& =\frac{1}{(N-1)^{2}} \mathbb{E}\left[\mathbb{E}\left[\left(\sum_{j \neq i}^{N} Z_{j}^{i}\right)\left(\sum_{k \neq i}^{N} Z_{k}^{i}\right) \mid \mathcal{F}_{t}^{W}, Y_{s}^{i, \varepsilon}\right]\right] \\
& =\frac{1}{(N-1)^{2}} \mathbb{E}\left[\mathbb{E}\left[\sum_{j \neq i}^{N}\left|Z_{j}^{i}\right|^{2} \mid \mathcal{F}_{t}^{W}, Y_{s}^{i, \varepsilon}\right]\right]=\frac{1}{N-1} \mathbb{E}\left[\left|Z_{1}^{2}\right|^{2}\right] .
\end{aligned}
$$


Due to the fact that, using (2.3),

$\mathbb{E}\left[\left|Z_{2}^{1}\right|^{2}\right]=\mathbb{E}\left[\left(\nabla \mathcal{G}_{\varepsilon}\left(Y_{s}^{1, \varepsilon}-Y_{s}^{2, \varepsilon}\right)-\nabla \mathcal{G}_{\varepsilon} * \rho_{s}^{\varepsilon}\left(Y_{s}^{1, \varepsilon}\right)\right)^{2}\right] \leq 4\left\|\nabla \mathcal{G}_{\varepsilon}\right\|_{\infty}^{2} \leq C \varepsilon^{-2(d-1)}$

one has

$$
\mathbb{E}\left[\left|I_{12}^{S}\right|^{2}\right] \leq \frac{C \varepsilon^{-2(d-1)}}{N-1}
$$

Thus, we concludes

$$
\begin{aligned}
& \mathbb{E}\left[\int_{0}^{t} 2 \chi\left(X_{s}^{i, \varepsilon}-Y_{s}^{i, \varepsilon}\right) \cdot I_{12}^{s} \mathrm{~d} s\right] \\
& \quad \leq \int_{0}^{t} \mathbb{E}\left[\left|X_{s}^{i, \varepsilon}-Y_{s}^{i, \varepsilon}\right|^{2}\right] \mathrm{d} s+\int_{0}^{t} \chi^{2} \mathbb{E}\left[\left|I_{12}^{s}\right|^{2}\right] \mathrm{d} s \leq \int_{0}^{t} \mathbb{E}\left[\left|X_{s}^{i, \varepsilon}-Y_{s}^{i, \varepsilon}\right|^{2}\right] \mathrm{d} s \\
& \quad+\frac{C \varepsilon^{-2(d-1)}}{N-1}
\end{aligned}
$$

where $C$ depends only on $\chi$ and $d$.

Now, collecting estimates (5.12) and (5.13) implies

$$
\begin{aligned}
& \mathbb{E}\left[\int_{0}^{t} 2 \chi\left(X_{s}^{i, \varepsilon}-Y_{s}^{i, \varepsilon}\right) \cdot\left(\frac{1}{N-1} \sum_{j \neq i}^{N} \nabla \mathcal{G}_{\varepsilon}\left(X_{s}^{i, \varepsilon}-X_{s}^{j, \varepsilon}\right)-\nabla \mathcal{G}_{\varepsilon} * \rho_{s}^{\varepsilon}\left(Y_{s}^{i, \varepsilon}\right)\right) \mathrm{d} s\right] \\
& \leq C \varepsilon^{-d} \int_{0}^{t} \sup _{i=1, \ldots, N} \mathbb{E}\left[\left|X_{s}^{i, \varepsilon}-Y_{s}^{i, \varepsilon}\right|^{2}\right] \mathrm{d} s+\frac{C \varepsilon^{-2(d-1)}}{N-1}
\end{aligned}
$$

which together with (5.11) lead to

$$
\sup _{i=1, \ldots, N} \mathbb{E}\left[\left|X_{t}^{i, \varepsilon}-Y_{t}^{i, \varepsilon}\right|^{2}\right] \leq C_{1} \varepsilon^{-d} \int_{0}^{t} \sup _{i=1, \ldots, N} \mathbb{E}\left[\left|X_{s}^{i, \varepsilon}-Y_{s}^{i, \varepsilon}\right|^{2}\right] \mathrm{d} s+\frac{C_{2} \varepsilon^{-2(d-1)}}{N-1}
$$

Applying Gronwall's inequality further yields that

$$
\sup _{t \in[0, T]} \sup _{i=1, \ldots, N} \mathbb{E}\left[\left|X_{t}^{i, \varepsilon}-Y_{t}^{i}\right|^{2}\right] \leq \frac{C_{2} \varepsilon^{-2(d-1)}}{N-1} e^{C_{1} \varepsilon^{-d} T} \leq C \frac{(\delta \ln (N))^{\frac{2 d-2}{d}}}{N^{1-C \delta}}
$$

where we let $e^{\varepsilon^{-d}} \leq N^{\delta}$, i.e., $\varepsilon^{-d} \leq \delta \ln (N)$, for any fixed $0<\delta<\frac{1}{C}$. The proof is completed.

Theorem 5.1 implies the convergence in law of the empirical measure in the following sense: 
Corollary 2 Under the same assumptions as in Theorem 5.1, the empirical measure

$$
\rho_{t}^{\varepsilon, N}:=\frac{1}{N} \sum_{i=1}^{N} \delta_{X_{t}^{i, \varepsilon}}
$$

associated with the stochastic particle system (1.5) converges weakly to unique solution $\rho_{t}$ to the nonlinear SPDE (1.3). More precisely, for any fixed $0<\delta \ll 1$, such that $\varepsilon^{-d}=\delta \ln (N)$ and $C \delta<1$, it holds that for all $t \in[0, T]$

$$
\mathbb{E}\left[\left|\left\langle\rho_{t}^{\varepsilon, N}, \phi\right\rangle-\left\langle\rho_{t}, \phi\right\rangle\right|^{2}\right] \leq C\left(\frac{(\delta \ln (N))^{\frac{2 d-2}{d}}}{N^{1-C \delta}}+\frac{1}{N}+(\delta \ln (N))^{-\frac{2}{d}}\right),
$$

for any $\phi \in C_{c}^{1}\left(\mathbb{R}^{d}\right)$, where $C$ depends only on $\|\phi\|_{C^{1}}, \chi, T, \lambda, \Lambda, d$, and $\left\|\rho_{0}\right\|_{W^{2,2}\left(\mathbb{R}^{d}\right)}$.

Proof Let us compute

$$
\begin{aligned}
& \mathbb{E}\left[\left|\left\langle\rho_{t}^{\varepsilon, N}, \phi\right\rangle-\left\langle\rho_{t}^{\varepsilon}, \phi\right\rangle\right|^{2}\right] \\
& =\mathbb{E}\left[\left|\frac{1}{N} \sum_{i=1}^{N} \phi\left(X_{t}^{i, \varepsilon}\right)-\int_{\mathbb{R}^{d}} \phi(x) \rho_{t}^{\varepsilon}(x) \mathrm{d} x\right|^{2}\right] \\
& \leq 2 \mathbb{E}\left[\left|\phi\left(X_{t}^{1, \varepsilon}\right)-\phi\left(Y_{t}^{1, \varepsilon}\right)\right|^{2}\right]+2 \mathbb{E}\left[\left|\frac{1}{N} \sum_{i=1}^{N} \phi\left(Y_{t}^{i, \varepsilon}\right)-\int_{\mathbb{R}^{d}} \phi(x) \rho_{t}^{\varepsilon}(x) \mathrm{d} x\right|^{2}\right] \\
& =: I_{1}+I_{2} .
\end{aligned}
$$

According to (5.10), one has

$$
\begin{aligned}
I_{1} & \leq 2\|\nabla \phi\|_{\infty}^{2} \mathbb{E}\left[\left|X_{t}^{1, \varepsilon}-Y_{t}^{1, \varepsilon}\right|^{2}\right] \\
& \leq C \frac{(\delta \ln (N))^{\frac{2 d-2}{d}}}{N^{1-C \delta}}
\end{aligned}
$$

where $C$ depends only on $\|\nabla \phi\|_{\infty}, \chi, T, \lambda, \Lambda, d$ and $\left\|\rho_{0}\right\|_{W^{2,2}\left(\mathbb{R}^{d}\right)}$. To estimate $I_{2}$, we compute that

$$
\begin{aligned}
& \mathbb{E}\left[\left|\frac{1}{N} \sum_{i=1}^{N} \phi\left(Y_{t}^{i, \varepsilon}\right)-\int_{\mathbb{R}^{d}} \phi(x) \rho_{t}^{\varepsilon}(x) \mathrm{d} x\right|^{2}\right] \\
& \leq \frac{1}{N^{2}} \sum_{i=1}^{N} \mathbb{E}\left[\left|\phi\left(Y_{t}^{i, \varepsilon}\right)-\int_{\mathbb{R}^{d}} \phi(x) \rho_{t}^{\varepsilon}(x)\right|^{2}\right] \leq C \frac{1}{N},
\end{aligned}
$$


where $C$ depends only on $\|\phi\|_{\infty}$. This combined with (5.17) implies

$$
\mathbb{E}\left[\left|\left\langle\rho_{t}^{\varepsilon, N}, \phi\right\rangle-\left\langle\rho_{t}^{\varepsilon}, \phi\right\rangle\right|^{2}\right] \leq C\left(\frac{(\delta \ln (N))^{\frac{2 d-2}{d}}}{N^{1-C \delta}}+\frac{1}{N}\right)
$$

Next, using (5.9) we compute

$$
\mathbb{E}\left[\left|\left\langle\rho_{t}^{\varepsilon}, \phi\right\rangle-\left\langle\rho_{t}, \phi\right\rangle\right|^{2}\right] \leq C \mathbb{E}\left[\sup _{t \in[0, T]}\left\|\rho_{t}^{\varepsilon}-\rho_{t}\right\|_{4}^{2}\right]=C\left\|\rho^{\varepsilon}-\rho\right\|_{S_{\mathcal{F} W}^{2}\left([0, T] ; L^{4}\left(\mathbb{R}^{d}\right)\right)}^{2} \leq C \varepsilon^{2} .
$$

Hence, one has

$$
\begin{aligned}
\mathbb{E}\left[\left|\left\langle\rho_{t}^{\varepsilon, N}, \phi\right\rangle-\left\langle\rho_{t}, \phi\right\rangle\right|^{2}\right] & \leq 2 \mathbb{E}\left[\left|\left\langle\rho_{t}^{\varepsilon, N}, \phi\right\rangle-\left\langle\rho_{t}^{\varepsilon}, \phi\right\rangle\right|^{2}\right]+2 \mathbb{E}\left[\left|\left\langle\rho_{t}^{\varepsilon}, \phi\right\rangle-\left\langle\rho_{t}, \phi\right\rangle\right|^{2}\right] \\
& \leq C\left(\frac{(\delta \ln (N))^{\frac{2 d-2}{d}}}{N^{1-C \delta}}+\frac{1}{N}+(\delta \ln (N))^{-\frac{2}{d}}\right) .
\end{aligned}
$$

This completes the proof.

Funding Open Access funding enabled and organized by Projekt DEAL.

Open Access This article is licensed under a Creative Commons Attribution 4.0 International License, which permits use, sharing, adaptation, distribution and reproduction in any medium or format, as long as you give appropriate credit to the original author(s) and the source, provide a link to the Creative Commons licence, and indicate if changes were made. The images or other third party material in this article are included in the article's Creative Commons licence, unless indicated otherwise in a credit line to the material. If material is not included in the article's Creative Commons licence and your intended use is not permitted by statutory regulation or exceeds the permitted use, you will need to obtain permission directly from the copyright holder. To view a copy of this licence, visit http://creativecommons.org/licenses/by/4.0/.

\section{References}

Bellomo, N., Bellouquid, A., Chouhad, N.: From a multiscale derivation of nonlinear cross-diffusion models to Keller-Segel models in a Navier-Stokes fluid. Math. Models Methods Appl. Sci. 26(11), 2041-2069 (2016)

Bensoussan, A., Frehse, J., Yam, P.: Mean Field Games and Mean Field Type Control Theory. Springer, Berlin (2013)

Biler, P.: Mathematical challenges in the theory of chemotaxis. Sci. Ann. Math. Sil. 32, 43-63 (2018)

Biler, P., Karch, G.: Blowup of solutions to generalized Keller-Segel model. J. Evol. Equ. 10(2), 247-262 (2010)

Blanchet, A., Dolbeault, J., Perthame, B.: Two-dimensional Keller-Segel model: optimal critical mass and qualitative properties of the solutions, 2006. Electron. J. Differ. Equ. (EJDE) [electronic only] 44, 1-33 (2006)

Blanchet, A., Carrillo, J.A., Masmoudi, N.: Infinite time aggregation for the critical Patlak-Keller-Segel model in $\mathbb{R}^{2}$. Commun. Pure Appl. Math. 61(10), 1449-1481 (2008)

Bresch, D., Jabin, P.-E., Wang, Z.: On mean-field limits and quantitative estimates with a large class of singular kernels: application to the Patlak-Keller-Segel model. C. R. Math. 357(9), 708-720 (2019)

Brezis, H.: Functional Analysis. Sobolev Spaces and Partial Differential Equations. Springer, Berlin (2010)

Brzezniak, Z., Flandoli, F., Maurelli, M.: Existence and uniqueness for stochastic 2D Euler flows with bounded vorticity. Arch. Ration. Mech. Anal. 221(1), 107-142 (2016) 
Burini, D., Chouhad, N.: A multiscale view of nonlinear diffusion in biology: from cells to tissues. Math. Models Methods Appl. Sci. 29(04), 791-823 (2019)

Carmona, R., Delarue, F.: Probabilistic Theory of Mean Field Games with Applications I-II. Springer, Berlin (2018)

Carmona, R., Delarue, F., Lacker, D.: Mean field games with common noise. Ann. Probab. 44(6), 3740-3803 (2016)

Carrillo, J.A., Choi, Y.-P., Hauray, M.: The derivation of swarming models: mean-field limit and Wasserstein distances. Collective Dyn. Bacteria Crowds 553, 1-46 (2014)

Cattiaux, P., Pédèches, L.: The 2-d stochastic Keller-Segel particle model: existence and uniqueness (2016). arXiv preprint arXiv:1601.08026

Choi, Y.-P., Salem, S.: Cucker-Smale flocking particles with multiplicative noises: stochastic mean-field limit and phase transition. Kinetic Relat. Models 12(3), 573-592 (2019)

Coghi, M., Flandoli, F.: Propagation of chaos for interacting particles subject to environmental noise. Ann. Appl. Probab. 26(3), 1407-1442 (2016)

Corrias, L., Perthame, B., Zaag, H.: Global solutions of some chemotaxis and angiogenesis systems in high space dimensions. Milan J. Math. 72(1), 1-28 (2004)

Dolbeault, J., Perthame, B.: Optimal critical mass in the two dimensional Keller-Segel model in $\mathbb{R}^{2}$. C. R. Math. 339(9), 611-616 (2004)

Du, K., Qiu, J., Tang, S.: $L^{p}$ theory for super-parabolic backward stochastic partial differential equations in the whole space. Appl. Math. Optim. 65(2), 175-219 (2011)

Escudero, C.: The fractional Keller-Segel model. Nonlinearity 19(12), 2909 (2006)

Fetecau, R.C., Huang, H., Sun, W.: Propagation of chaos for the Keller-Segel equation over bounded domains. J. Differ. Equ. 266(4), 2142-2174 (2019)

Fournier, N., Jourdain, B.: Stochastic particle approximation of the Keller-Segel equation and twodimensional generalization of Bessel processes. Ann. Appl. Probab. 27(5), 2807-2861 (2017)

Haškovec, J., Schmeiser, C.: Convergence of a stochastic particle approximation for measure solutions of the 2d Keller-Segel system. Commun. Partial Differ. Equ. 36(6), 940-960 (2011)

Hilbert, D.: Mathematical problems. Bull. Am. Math. Soc. 8(10), 437-479 (1902)

Hillen, T., Painter, K.J.: A user's guide to PDE models for chemotaxis. J. Math. Biol. 58(1-2), 183 (2009)

Horstmann, D.: From 1970 Until Present: The Keller-Segel Model in Chemotaxis and Its Consequences (2003)

Huang, H., Liu, J.-G.: Well-posedness for the Keller-Segel equation with fractional Laplacian and the theory of propagation of chaos. Kinet. Relat. Models 9(4), 715-748 (2016)

Huang, H., Liu, J.-G.: Discrete-in-time random particle blob method for the Keller-Segel equation and convergence analysis. Commun. Math. Sci. 15(7), 1821-1842 (2017a)

Huang, H., Liu, J.-G.: Error estimate of a random particle blob method for the Keller-Segel equation. Math. Comput. 86(308), 2719-2744 (2017b)

Huang, H., Liu, J.-G., Lu, J.: Learning interacting particle systems: diffusion parameter estimation for aggregation equations. Math. Models Methods Appl. Sci. 29(01), 1-29 (2019)

Jabin, P.-E., Wang, Z.: Mean Field Limit for Stochastic Particle Systems, Active Particles, vol. 1, pp. 379-402. Springer, Berlin (2017)

Jäger, W., Luckhaus, S.: On explosions of solutions to a system of partial differential equations modelling chemotaxis. Trans. Am. Math. Soc. 329(2), 819-824 (1992)

Karatzas, I., Shreve, S.E.: Brownian Motion and Stochastic Calculus. Springer, Berlin (1998)

Keller, E.F., Segel, L.A.: Initiation of slime mold aggregation viewed as an instability. J. Theor. Biol. 26(3), 399-415 (1970)

Krylov, N.V.: An analytic approach to SPDEs, Stochastic partial differential equations: six perspectives. AMS Math. Surv. Monogr. 64, 185-242 (1999)

Krylov, N.V.: Itô's formula for the $L_{p}$-norm of stochastic $W_{p}^{1}$-valued processes. Probab. Theory Relat. Fields 147(3), 583-605 (2010)

Perthame, B.: Transport Equations in Biology. Springer, Berlin (2006)

Ren, J., Röckner, M., Wang, F.: Stochastic generalized porous media and fast diffusion equations. J. Differ. Equ. 238(1), 118-152 (2007)

Triebel, H.: Theory of Function Spaces. Birkhäuser, Basel (1983)

Yang, Z., Tang, S.: Dynkin game of stochastic differential equations with random coefficients, and associated backward stochastic partial differential variational inequality. SIAM J. Control Optim. 51(1), 64-95 (2013) 
Zhou, X.: A duality analysis on stochastic partial differential equations. J. Funct. Anal. 103, 275-293 (1992)

Publisher's Note Springer Nature remains neutral with regard to jurisdictional claims in published maps and institutional affiliations. 